\title{
Spectroscopic Behavior, FMO, NBO Analysis of Pethidine and Diclofenac Drugs by Theoretical Approach
}

\author{
Mostafa Khajehzadeha,b,* iD, Sedigheh Rahmaniaslc \\ a Department of Chemistry, Faculty of Science, Arak Branch, Islamic Azad University, Arak, Iran \\ b Young Researchers and Elite Club, Gachsaran Branch, Islamic Azad University, Gachsaran, Iran \\ c Department of Chemistry, Faculty of Science, Gachsaran Branch, Islamic Azad University, Gachsaran, Iran
}

\section{ARTICLE IN F O}

Received: 30 August 2019

Revised: 13 October 2019

Accepted: 15 November 2019

Available online: 04 December 2019

\section{K E Y W O R D S}

Pethidine
Diclofenac
UV-vis
NMR
FT-IR
Solvent effect
NBO
HOMO
LUMO
Electro negativity
Electrophilicity index
Chemical potential

\section{A B S T R A C T}

In this work, the complete structural, vibrational, electronic, and spectroscopic properties $\left({ }^{1} \mathrm{H},{ }^{13} \mathrm{C} N \mathrm{NR}, \mathrm{UV}\right.$-vis) and, natural bond orbital (NBO), Frontier molecular orbital (FMO) analysis of ethyl,1-methyl-4phenylpiperidine-4-carboxylate (pethidine) and 2-(2- $((2,6-$ dichlorophenyl)amino)phenyl)acetic acid (diclofenac) drugs were investigated in the gas and liquid phases by using the density functional theory (DFT/B3PW91) method and DGDZVP level of theory. Moreover, CIS-DGDZVP was used to calculate the energy and wavelength absorption $\left(\lambda_{\max }\right)$ of electronic transitions and its nature within the pethidine and diclofenac drugs. Therefore, for further analysis of these drugs, the effects of solvents on UV-vis and NMR spectra were investigated. The results revealed that the polarity of the solvents plays a crucial role in the structure and properties of the pethidine and diclofenac drugs. The ${ }^{1} \mathrm{H}$ and ${ }^{13} \mathrm{C}$ NMR spectra, NBO, the amount of global hardness, softness, ionization energy, electron affinity energy, the highest occupied molecular orbital (HOMO), lowest unoccupied molecular orbital (LUMO), Frontier molecular orbitals analysis, hybridization, zero-point energy (ZPE), total energy $\left(E_{\mathrm{T}}\right)$, Dipole Moment $(m)$, polarizability $(\alpha)$, MEP, bond lengths, bond angles, and electro negativity were calculated in the gas and liquid phases. 3D-plots of the molecular electrostatic potential (MESP) for the studied compounds were investigated and analyzed to assess the distribution of electronic density of orbitals and nucleophilic sites of the selected molecules. The results of the spectra showed that the solvents had greater effects on pethidine. Nevertheless, as for $\mathrm{CH}_{3} \mathrm{OH}$ solvent, the zero-point energy equals to 0.344085 , the total energy equal to $226.673 \mathrm{kcal} / \mathrm{mol}$ and dipole moment equal to 2.520 a.u were produced.

* Corresponding author's E-mail address: mkhajehzadeh93@iau-arak.ac.ir 


\section{GRA P H I C A L A B S TRACT}

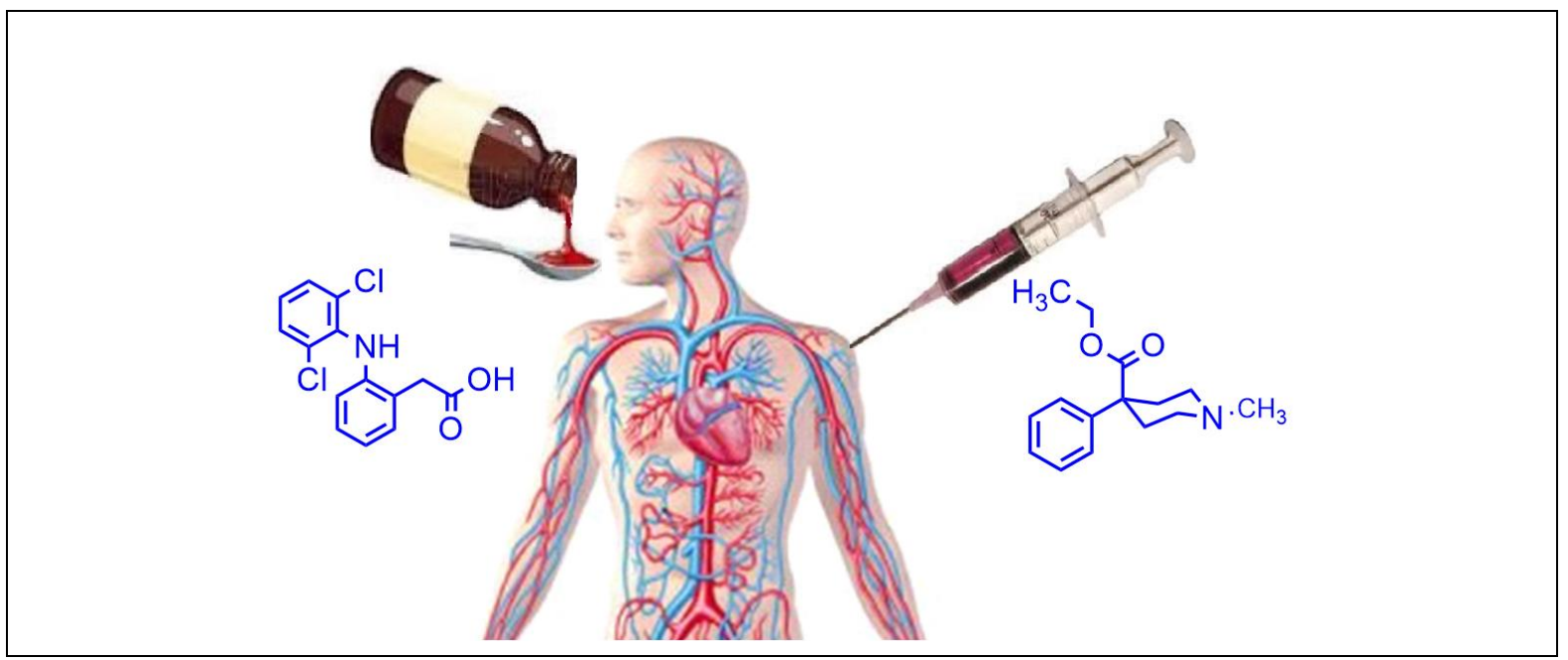

\section{Introduction}

Pethidine (ethyl,1-methyl-4-phenylpiperidine-4-carboxylate), known as meperidine or Demerol, is a synthetic opioid analgesic frequently prescribed in the emergency department. It was synthesized in 1939 as a potential anticholinergic agent. Meperidine is often a preferred analgesic drug used to relieve migraine headaches. Meperidine is indicated for the treatment of moderate to severe pain, and is delivered as syrup, intramuscular, and intravenous injection $[1,2]$. Meperidine is a phenylpiperidine derivative, which is firstly synthetic analgesic drug to achieve the wide therapeutic usage $[3,4]$. Meperidine has the similar pharmacological effect with morphine. However, meperidine was found to be safer than morphine, carrying a lower risk of addiction, treating the pain associated with biliary spasm and renal colic due to its putative anticholinergic effects [5]. A series of pethidine analogs were synthesized and their affinities for the $\left[{ }^{3} \mathrm{H}\right] \mathrm{N}$-methyl-scopolamine (NMS) binding site on muscarinic acetylcholine receptors were determined [6]. Pethidine has been also used to relieve labor pain [7]. Also, a method for the quantitative determination of pethidine (meperidine) and its metabolic norpethidine (normeperidine) in human hair by LC-MS-MS has been proposed [8].

Diclofenac is a lipophilic, nonsteroidal antiinflammatory drug that has been frequently used in human and animal health care to treat pain in musculoskeletal injuries and rheumatoid with an estimated annual consumption of several hundred tons [9]. Diclofenac is reported to covalently bind to macromolecules in situations where intracellular levels of NADH, NADPH, GSH, and other reducing agents are low. Also, diclofenac is often used to treat chronic pain associated with cancer, in particular if inflammation is also present $[10,11]$. Most recently, many studies have been conducted on the drugs by using computational methods. Quantum mechanics is a useful and popular method in chemistry and many studies in various fields [12-18]. Diclofenac is known to be a persistent pharmaceutical compound, and their effective removal from water sources has been a rising concern [19]. Diclofenac capsules have been released in many PEObased polymeric micelles as a shell and different polyesters as hydrophobic nuclei [20].

The objective of the present study aims at calculating the density functional theory 
(DFT/B3PW91) method and DGDZVP level of theory to obtain geometries, electronic structures, natural bonding orbital's (NBO), FTIR, NMR and UV-vis spectra, ionization energy, electron affinity, electro negativity and molecular electrostatic potential contours of pethidine and diclofenac drugs, these parameters characterize the forces that govern the structure of studied compounds. The hybridization of each atom, natural charges, bonding and antibonding orbital's second order perturbation energy $\left(E^{2}\right)$, exact configurations and Lewis and non-Lewis electrons results from natural bonding orbital analysis (Scheme 1).

\section{Experimental}

\section{Computational details}

The entire calculations were performed using the DFT/B3PW91 method and DGDZVP level of theory by the Gaussian 09 W suite software [21]. Following the geometry optimizations of pethidine and diclofenac drugs with the B3PW91 method, the optimized structural parameters were used in the vibrational wavenumber calculation to characterize all the stationary points while minima [22]. Energy gap and hybridization bonds, type of electron transfers between levels, natural atomic charge, and the amount of global hardness and global softness were calculated using the natural bond orbital (NBO) analysis at the presence of the solvents (such as $\mathrm{H}_{2} \mathrm{O}$, DMSO, $\mathrm{CH}_{3} \mathrm{CN}, \mathrm{C}_{3} \mathrm{H}_{6} \mathrm{O}, \mathrm{C}_{2} \mathrm{H}_{5} \mathrm{OH}$ and $\mathrm{CH}_{3} \mathrm{OH}$ ) on the optimized structure [23]. The electronic transition properties including the maximum excitation wavelength $\left(\lambda_{\max }\right)$, relative intensities (oscillator strengths, f), Mulliken charges, and natural population analysis were calculated using the B3PW91 and (CIS-DFT) with DGDZVP level of theory at the presence of six solvents. Proton and carbon NMR signal peaks have been assigned using GIAO-B3LYP/DGDZVP method [24].

\section{Results and discussion}

\section{Optimized structure}

The bond lengths and bond angles corresponding to the optimized geometry of pethidine and diclofenac drugs were obtained using the (DFT/B3PW91) method and DGDZVP level of theory in the gas phase. The optimized geometry of the title compounds is illustrated in Figure 1. The calculated values of some significant structural parameters are summarized in Table 1.

As seen in Table 1, the $\mathrm{C}-\mathrm{C}$ bond lengths in the benzene ring in the optimized geometry of pethidine calculated at B3PW91/DGDZVP fall in the range: $1.399-1.408 \AA$.

Scheme 1. Pethidine and diclofenac drugs

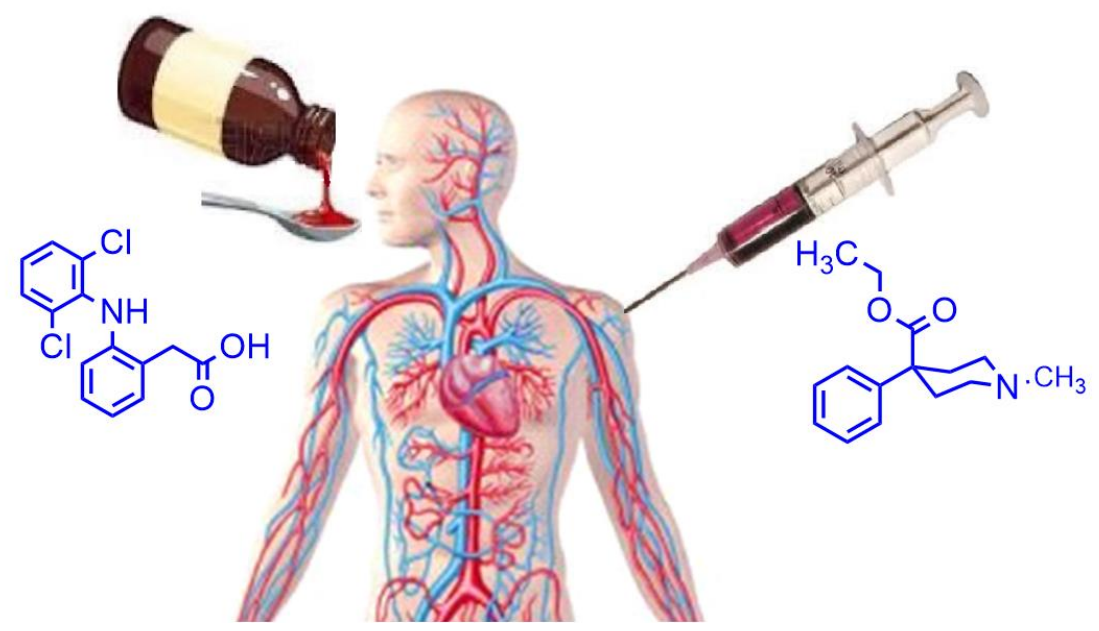


Figure 1. Molecular structure of pethidine and diclofenac drugs with atom numbering scheme

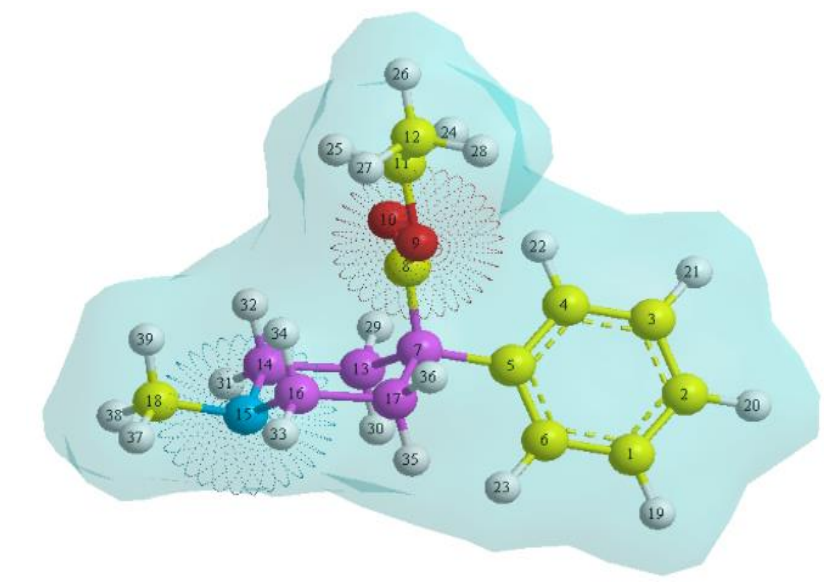

Pethidine

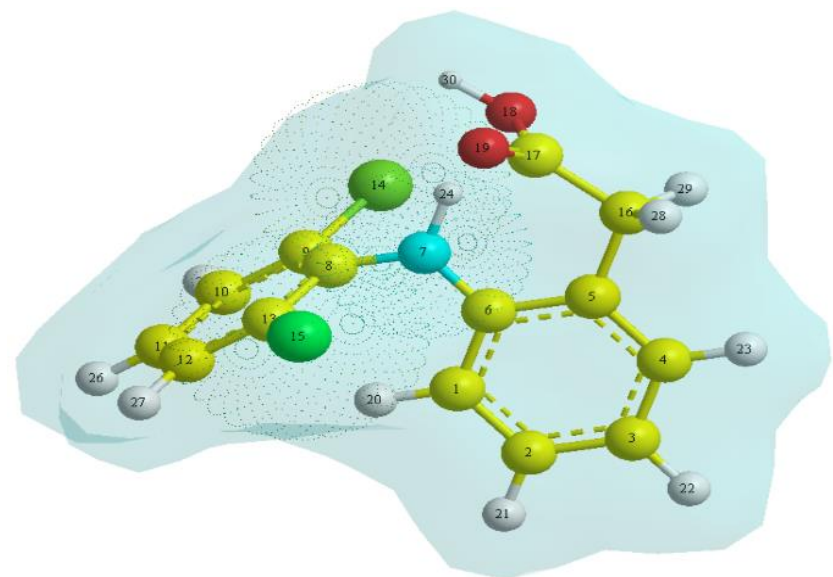

Diclofenac

Table 1. Optimized geometrical parameters of pethidine and diclofenac drugs by B3PW91/DGDZVP in the gas phase

\begin{tabular}{ccc|c}
$\begin{array}{c}\text { Parameter } \\
\text { Bond lengths }(\dot{\mathrm{A}})\end{array}$ & Pethidine & $\begin{array}{c}\text { Parameter } \\
\text { Bond lengths }(\dot{\mathrm{A}})\end{array}$ & Diclofenac \\
$\mathrm{C}_{1}-\mathrm{C}_{2}$ & 1.399 & $\mathrm{C}_{1}-\mathrm{C}_{2}$ & 1.397 \\
$\mathrm{C}_{4}-\mathrm{C}_{5}$ & 1.408 & $\mathrm{C}_{6}-\mathrm{N}_{7}$ & 1.419 \\
$\mathrm{C}_{5}-\mathrm{C}_{7}$ & 1.542 & $\mathrm{~N}_{7}-\mathrm{C}_{8}$ & 1.398 \\
$\mathrm{C}_{7}-\mathrm{C}_{8}$ & 1.533 & $\mathrm{C}_{9}-\mathrm{Cl}_{14}$ & 1.809 \\
$\mathrm{C}_{8}-\mathrm{C}_{9}$ & 1.367 & $\mathrm{C}_{13}-\mathrm{Cl}_{15}$ & 1.801 \\
$\mathrm{C}_{8}-\mathrm{O}_{10}$ & 1.239 & $\mathrm{C}_{17}-\mathrm{O}_{18}$ & 1.389 \\
$\mathrm{C}_{14}-\mathrm{C}_{15}$ & 1.470 & $\mathrm{C}_{17}-\mathrm{O}_{19}$ & 1.229 \\
$\left.\mathrm{Bond}_{\text {angles }}{ }^{\circ}\right)$ & & $\mathrm{Bond}_{1}$ angles & \\
$\left.\mathrm{C}_{4}-\mathrm{C}_{5}-\mathrm{C}_{6}\right)$ & \\
$\mathrm{C}_{5}-\mathrm{C}_{7}-\mathrm{C}_{8}$ & 118.380 & $\mathrm{C}_{4}-\mathrm{C}_{5}-\mathrm{C}_{6}$ & 119.132 \\
$\mathrm{C}_{7}-\mathrm{C}_{8}-\mathrm{O}_{9}$ & 105.600 & $\mathrm{C}_{6}-\mathrm{N}_{7}-\mathrm{C}_{8}$ & 124.859 \\
$\mathrm{C}_{7}-\mathrm{C}_{8}-\mathrm{O}_{10}$ & $\mathrm{C}_{8}-\mathrm{C}_{9}-\mathrm{Cl}_{14}$ & 118.635 \\
$\mathrm{C}_{8}-\mathrm{O}_{9}-\mathrm{C}_{11}$ & $\mathrm{C}_{8}-\mathrm{C}_{13}-\mathrm{Cl}_{15}$ & 119.840 \\
$\mathrm{C}_{10}-\mathrm{C}_{11}-\mathrm{C}_{12}$ & 112.849 & $\mathrm{O}_{18}-\mathrm{C}_{17}-\mathrm{O}_{19}$ & 121.174 \\
$\mathrm{C}_{14}-\mathrm{N}_{15}-\mathrm{C}_{16}$ & 125.327 & $\mathrm{C}_{5}-\mathrm{C}_{16}-\mathrm{C}_{17}$ & 112.799 \\
$\mathrm{C}_{14}-\mathrm{N}_{15}-\mathrm{C}_{18}$ & 117.587 & $\mathrm{C}_{6}-\mathrm{N}_{7}-\mathrm{H}_{24}$ & 114.382 \\
\hline
\end{tabular}


However, $\mathrm{C}-\mathrm{C}$ bond lengths in the benzene ring of diclofenac was calculated in the area: $1.397 \AA$. Also, C=0 bond lengths in the optimized geometry of pethidine was calculated in the area: $1.239 \AA$. But, $\mathrm{C}=0$ bond lengths of diclofenac was calculated in the area: $1.229 \AA$. Also, $\mathrm{C}-0$ bond lengths of pethidine was calculated in the area: 1.367 $\AA$. But, $\mathrm{C}-\mathrm{O}$ bond lengths of diclofenac was calculated in the area: $1.389 \AA$. Based on these results, $\mathrm{C}-\mathrm{C}$ and $\mathrm{C}=\mathrm{O}$ bond lengths in the optimized geometry of pethidine are greater than that of the diclofenac. However, $\mathrm{C}-\mathrm{O}$ bond lengths of diclofenac is larger than pethidine [25].

\section{FT-IR spectrum}

Figure 1 depicts the optimized molecular structure of pethidine and diclofenac drugs. Pethidine consists of 39 atoms, so it has 111 normal vibrational modes with the molecular formula of $\mathrm{C}_{15} \mathrm{H}_{21} \mathrm{NO}_{2}$ that belongs to the point group $\mathbf{C}_{\mathbf{1}}$ in its lowest energy case calculated at DGDZVP level of theory in the gas and liquid phases. On the other hand, diclofenac consists of 30 atoms, so it has 84 normal vibrational modes with the molecular formula of $\mathrm{C}_{14} \mathrm{H}_{11} \mathrm{Cl}_{2} \mathrm{NO}_{2}$, belonging to the point group $\mathbf{C}_{\boldsymbol{1}}$ in its lowest energy case calculated at DGDZVP level of theory in the gas and liquid phases. The molecular thermal enthalpies generated by Gaussian 09 are particularly dependent on the accuracy of the vibrational frequencies. The thermal term of the internal energy $\left(E_{T}\right)$, is composed of vibrational, rotational and translational energies. This study assesses the performance of quantum chemical models with regard to the calculation of zero-point energy, total energy, Dipole Moment and polarizability of pethidine and diclofenac drugs in various solvents (Table 2). It was found that, the total energy calculated at the presence of solvent for pethidine was found to be greater than the diclofenac. However, the calculated polarization for the diclofenac was higher compared with that of the pethidine.

To obtain the spectroscopic signature of pethidine and diclofenac drugs, a frequency calculation analysis was performed. Harmonic vibrational frequencies of the compounds were obtained using the B3PW91/DGDZVP (as seen in Figure 2). The wave-numbers of the IR-active are scaled by 0.961 , and which are shown with (vstretching, $\pi$-in plane bending, $\alpha$-out of plane bending) $[26,27]$.

FT-IR spectrum data for pethidine drug calculated by B3PW91/DGDZVP

Gas phase

IR $\left(\mathrm{cm}^{-1}\right): \boldsymbol{v}=3139,3125,3119,3069$, 3004, $2963(\mathrm{C}-\mathrm{H}), 1637,1626(\mathrm{C}=0), \pi=$ $1604(\mathrm{C}=\mathrm{C}), 1486,1479,1459,1445\left(\mathrm{CH}_{3}\right)$, 1206 (C-0), 1160 (C-C), $1152(\mathrm{C}-\mathrm{N}), 1098$, $1070(\mathrm{C}-\mathrm{H}), \boldsymbol{\alpha}=992,942,907,838(\mathrm{C}-\mathrm{H})$, $739(\mathrm{C}-0), 640,620(\mathrm{C}-\mathrm{C})$.

\section{Liquid phase}

IR $\left(\mathrm{cm}^{-1}, \mathbf{H}_{\mathbf{2}} \mathbf{0}\right): \boldsymbol{v}=3140,3126,3094$, 3056, $2848(\mathrm{C}-\mathrm{H}), 1632,1624(\mathrm{C}=0), \pi=$ 1604, 1603 ( $\mathrm{C}=\mathrm{C}), 1490,1481,1478,1439$ $\left(\mathrm{CH}_{3}\right), 1197(\mathrm{C}-\mathrm{O}), 1159(\mathrm{C}-\mathrm{C}), 1149(\mathrm{C}-\mathrm{N})$, 1098, $1082(\mathrm{C}-\mathrm{H}), \boldsymbol{\alpha}=959,941,907,873$ (C-H), $792(\mathrm{C}-\mathrm{O}), 619,535(\mathrm{C}-\mathrm{C})$.

IR $\left(\mathrm{cm}^{-1}, \mathbf{C H}_{\mathbf{3}} \mathbf{O H}\right): \boldsymbol{v}=3140,3126,3094$, 3051, $2857(\mathrm{C}-\mathrm{H}), 1632,1624(\mathrm{C}=0), \pi=$ $1603(\mathrm{C}=\mathrm{C}), 1481,1473,1437\left(\mathrm{CH}_{3}\right), 1196$ (C-O), 1158 (C-C), 1148 (C-N), 1061, 1033 $(\mathrm{C}-\mathrm{H}), \boldsymbol{\alpha}=958,928,906,840(\mathrm{C}-\mathrm{H}), 791$ (C-0), 639, $536(\mathrm{C}-\mathrm{C})$.

IR $\left(\mathrm{cm}^{-1}, \mathbf{C}_{\mathbf{2}} \mathbf{H}_{\mathbf{5}} \mathbf{O H}\right): \boldsymbol{v}=3140,3126,3112$, 3053, $2848(\mathrm{C}-\mathrm{H}), 1631,1623(\mathrm{C}=0), \boldsymbol{\pi}=$ $1602(\mathrm{C}=\mathrm{C}), 1480,1478,1464\left(\mathrm{CH}_{3}\right), 1198$ (C-0), 1159 (C-C), 1149 (C-N), 1071, 1032 $(\mathrm{C}-\mathrm{H}), \boldsymbol{\alpha}=998,941,928,820(\mathrm{C}-\mathrm{H}), 790$ (C-0), 637, 508 (C-C).

IR ( $\mathrm{cm}^{-1}$, DMSO): $\boldsymbol{v}=3140,3124,3101$, 3065, $2989(\mathrm{C}-\mathrm{H}), 1630,1621(\mathrm{C}=0), \pi=$ 
$1601(\mathrm{C}=\mathrm{C}), 1481,1476,1456\left(\mathrm{CH}_{3}\right), 1197$ (C-O), 1157 (C-C), 1149 (C-N), 1082, 1061
$(\mathrm{C}-\mathrm{H}), \boldsymbol{\alpha}=991,959,926,835(\mathrm{C}-\mathrm{H}), 792$ (C-0), 639, 528 (C-C).

Figure 2. Computed infrared spectrum of pethidine and diclofenac drugs in the gas phase by B3PW91/DGDZVP

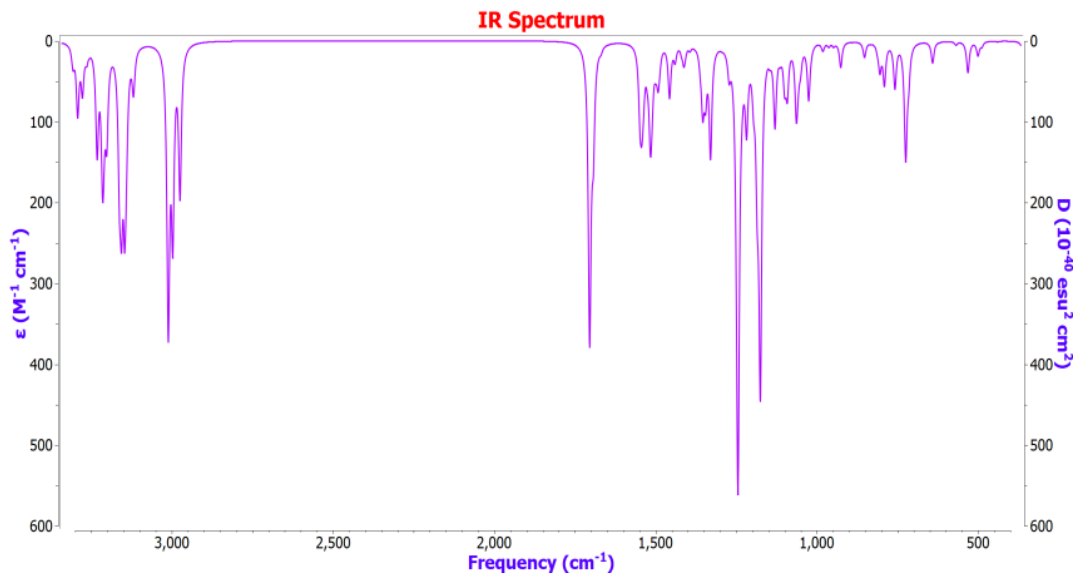

Pethidine

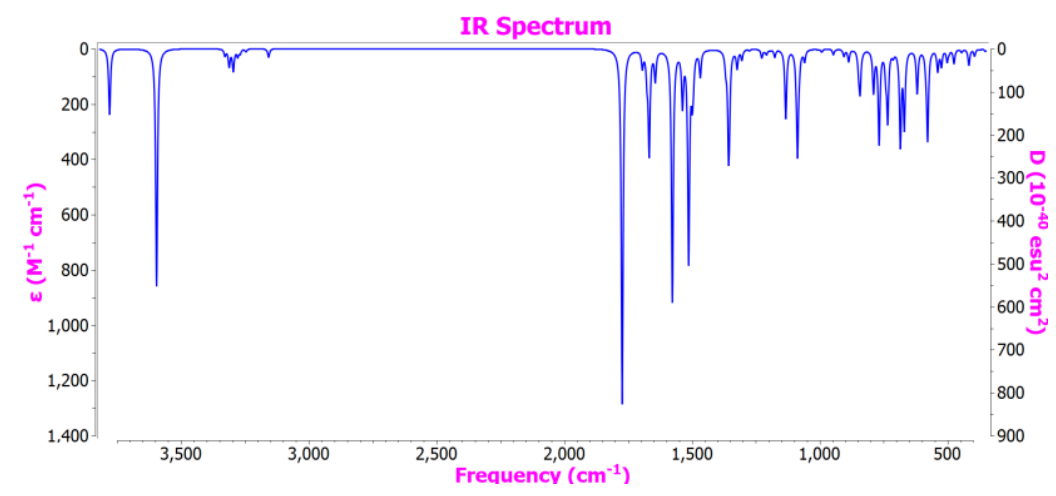

Diclofenac

IR $\left(\mathrm{cm}^{-1}, \mathbf{C H}_{\mathbf{3}} \mathbf{C N}\right): \boldsymbol{v}=3140,3125,3094$, 3053, $2987(\mathrm{C}-\mathrm{H}), 1632,1623(\mathrm{C}=0), \pi=$ $1602(\mathrm{C}=\mathrm{C}), 1490,1477,1464\left(\mathrm{CH}_{3}\right), 1197$ (C-0), $1159(\mathrm{C}-\mathrm{C}), 1148(\mathrm{C}-\mathrm{N}), 1098,1071$ $(\mathrm{C}-\mathrm{H}), \boldsymbol{\alpha}=998,941,928,820(\mathrm{C}-\mathrm{H}), 791$ (C-0), 638, $526(\mathrm{C}-\mathrm{C})$.

IR $\left(\mathrm{cm}^{-1}, \mathbf{C}_{\mathbf{3}} \mathbf{H}_{\mathbf{6}} \mathbf{0}\right): \boldsymbol{v}=3140,3124,3095$, 3051, $2986(\mathrm{C}-\mathrm{H}), 1631,1624(\mathrm{C}=0), \pi=$ 1603 (C=C), 1491, 1477, $1465\left(\mathrm{CH}_{3}\right), 1197$ (C-0), 1159 (C-C), 1149 (C-N), 1097, 1071 $(\mathrm{C}-\mathrm{H}), \boldsymbol{\alpha}=991,959,926,822(\mathrm{C}-\mathrm{H}), 790$ (C-0), 637, 529 (C-C).

FT-IR spectrum data for diclofenac drug calculated by B3PW91/DGDZVP

Gas phase
IR $\left(\mathrm{cm}^{-1}\right): \boldsymbol{v}=3569(\mathrm{O}-\mathrm{H}), 3397(\mathrm{~N}-\mathrm{H})$, 3149, 3135, 3133, 3121, 3118, 3102, 3094 $(\mathrm{C}-\mathrm{H}), 3072,2990\left(\mathrm{CH}_{2}\right), 1705(\mathrm{C}=0), \pi=$ 1632, 1615, 1607 (C=C), 1585, 1523, 1486 $(\mathrm{N}-\mathrm{H}), 1464(\mathrm{C}-\mathrm{N}), 1451,1447,1329,1319$ $\left(\mathrm{CH}_{2}\right), 1288(\mathrm{C}-0), 1073(\mathrm{C}-\mathrm{C}), \boldsymbol{\alpha}=995,980$ $(\mathrm{C}-\mathrm{H}), 938\left(\mathrm{CH}_{2}\right), 846(\mathrm{C}-\mathrm{O}), 841,741,680$ $(\mathrm{N}-\mathrm{H}), 602(\mathrm{C}-\mathrm{C})$.

\section{Liquid phase}

IR $\left(\mathrm{cm}^{-1}, \mathbf{H}_{\mathbf{2}} \mathbf{O}\right): \boldsymbol{v}=3567(\mathrm{O}-\mathrm{H}), 3391$ (N-H), 3150, 3137, 3133, 3124, 3119, 3104, $3096(\mathrm{C}-\mathrm{H}), 3073,2990\left(\mathrm{CH}_{2}\right), 1694(\mathrm{C}=0), \boldsymbol{\pi}$ $=1631,1614,1605(\mathrm{C}=\mathrm{C}), 1583,1517,1484$ $(\mathrm{N}-\mathrm{H}), 1460(\mathrm{C}-\mathrm{N}), 1449,1439,1319,1314$ $\left(\mathrm{CH}_{2}\right), 1286(\mathrm{C}-0), 1070(\mathrm{C}-\mathrm{Cl}), \boldsymbol{\alpha}=984,931$ $(\mathrm{C}-\mathrm{H}), 831\left(\mathrm{CH}_{2}\right), 689(\mathrm{C}-\mathrm{O}), 658,616(\mathrm{~N}-\mathrm{H})$, $515(\mathrm{C}-\mathrm{C})$. 
IR $\left(\mathrm{cm}^{-1}, \mathbf{C H}_{\mathbf{3}} \mathbf{O H}\right): \boldsymbol{v}=3566(\mathrm{O}-\mathrm{H}), 3390$ $(\mathrm{N}-\mathrm{H}), 3151,3138,3134,3125,3118,3104$, $3097(\mathrm{C}-\mathrm{H}), 3071,2990\left(\mathrm{CH}_{2}\right), 1692(\mathrm{C}=0), \boldsymbol{\pi}$ $=1630,1615,1604(\mathrm{C}=\mathrm{C}), 1581,1515,1482$ $(\mathrm{N}-\mathrm{H}), 1462(\mathrm{C}-\mathrm{N}), 1447,1438,1317,1312$ $\left(\mathrm{CH}_{2}\right), 1288(\mathrm{C}-0), 1065(\mathrm{C}-\mathrm{Cl}), \boldsymbol{\alpha}=980,927$ $(\mathrm{C}-\mathrm{H}), 830\left(\mathrm{CH}_{2}\right), 688(\mathrm{C}-\mathrm{O}), 654,610(\mathrm{~N}-\mathrm{H})$, $513(\mathrm{C}-\mathrm{C})$.

IR $\left(\mathrm{cm}^{-1}, \mathbf{C}_{\mathbf{2}} \mathbf{H}_{\mathbf{5}} \mathbf{O H}\right): \boldsymbol{v}=3567(\mathrm{O}-\mathrm{H}), 3391$ $(\mathrm{N}-\mathrm{H}), 3148,3136,3135,3126,3119,3105$, $3098(\mathrm{C}-\mathrm{H}), 3070,2990\left(\mathrm{CH}_{2}\right), 1692(\mathrm{C}=0), \pi$ $=1633,1616,1606(\mathrm{C}=\mathrm{C}), 1580,1517,1481$ $(\mathrm{N}-\mathrm{H}), 1461(\mathrm{C}-\mathrm{N}), 1449,1439,1318,1311$ $\left(\mathrm{CH}_{2}\right), 1289(\mathrm{C}-0), 1066(\mathrm{C}-\mathrm{Cl}), \boldsymbol{\alpha}=983,927$ $(\mathrm{C}-\mathrm{H}), 833\left(\mathrm{CH}_{2}\right), 688(\mathrm{C}-\mathrm{O}), 655,614(\mathrm{~N}-\mathrm{H})$, $515(\mathrm{C}-\mathrm{C})$.

IR ( $\mathrm{cm}^{-1}$, DMSO): $\boldsymbol{v}=3566(\mathrm{O}-\mathrm{H}), 3390$ $(\mathrm{N}-\mathrm{H}), 3150,3137,3133,3125,3119,3105$, $3096(\mathrm{C}-\mathrm{H}), 3072,2990\left(\mathrm{CH}_{2}\right), 1692(\mathrm{C}=0), \pi$ $=1631,1613,1605(\mathrm{C}=\mathrm{C}), 1583,1518,1481$ $(\mathrm{N}-\mathrm{H}), 1461(\mathrm{C}-\mathrm{N}), 1447,1437,1316,1314$ $\left(\mathrm{CH}_{2}\right), 1285(\mathrm{C}-0), 1063(\mathrm{C}-\mathrm{Cl}), \boldsymbol{\alpha}=983,930$ $(\mathrm{C}-\mathrm{H}), 833\left(\mathrm{CH}_{2}\right), 689(\mathrm{C}-\mathrm{O}), 658,617(\mathrm{~N}-\mathrm{H})$, $515(\mathrm{C}-\mathrm{C})$.

IR $\left(\mathrm{cm}^{-1}, \mathbf{C H}_{\mathbf{3}} \mathbf{C N}\right): \boldsymbol{v}=3567(\mathrm{O}-\mathrm{H}), 3390$ $(\mathrm{N}-\mathrm{H}), 3150,3136,3130,3123,3119,3105$, $3096(\mathrm{C}-\mathrm{H}), 3073,2990\left(\mathrm{CH}_{2}\right), 1694(\mathrm{C}=0), \pi$ $=1632,1614,1606(\mathrm{C}=\mathrm{C}), 1582,1518,1483$ $(\mathrm{N}-\mathrm{H}), 1461(\mathrm{C}-\mathrm{N}), 1448,1438,1319,1312$ $\left(\mathrm{CH}_{2}\right), 1286(\mathrm{C}-0), 1061(\mathrm{C}-\mathrm{Cl}), \boldsymbol{\alpha}=984,931$ $(\mathrm{C}-\mathrm{H}), 831\left(\mathrm{CH}_{2}\right), 688(\mathrm{C}-\mathrm{O}), 658,617(\mathrm{~N}-\mathrm{H})$, $514(\mathrm{C}-\mathrm{C})$.

IR $\left(\mathrm{cm}^{-1}, \mathbf{C}_{\mathbf{3}} \mathbf{H}_{\mathbf{6}} \mathbf{0}\right): \boldsymbol{v}=3567(\mathrm{O}-\mathrm{H}), 3392$ $(\mathrm{N}-\mathrm{H}), 3151,3135,3130,3122,3117,3102$, $3098(\mathrm{C}-\mathrm{H}), 3071,2991\left(\mathrm{CH}_{2}\right), 1693(\mathrm{C}=0), \pi$ $=1630,1615,1608(\mathrm{C}=\mathrm{C}), 1580,1517,1482$ $(\mathrm{N}-\mathrm{H}), 1460(\mathrm{C}-\mathrm{N}), 1449,1439,1318,1314$ $\left(\mathrm{CH}_{2}\right), 1285(\mathrm{C}-0), 1060(\mathrm{C}-\mathrm{Cl}), \boldsymbol{\alpha}=984,930$ $(\mathrm{C}-\mathrm{H}), 830\left(\mathrm{CH}_{2}\right), 687(\mathrm{C}-\mathrm{O}), 659,619(\mathrm{~N}-\mathrm{H})$, $516(\mathrm{C}-\mathrm{C})$.

\section{$U V$-vis spectrum analysis}

UV-vis analysis of pethidine and diclofenac drugs was evaluated using the theoretical calculation. The wavelength absorption $\left(\lambda_{\max }\right)$ for the pethidine and diclofenac drugs were calculated using the CIS-B3PW91 method with DGDZVP basis set in the gas and liquid phases (Figure 3), and the results are presented in Table 3.

The results revealed that, the max values obtained with CIS-B3PW91/DGDZVP for pethidine are $202.45 \mathrm{~nm}$ in the gas phase, and $200.86 \mathrm{~nm}$ in the liquid phase, and the lowest wavelength was calculated at the presence of $\mathrm{H}_{2} \mathrm{O}$ solvent. Also, the max values obtained with CIS-B3PW91/DGDZVP for diclofenac are $220.24 \mathrm{~nm}$ in the liquid phase (DMSO solvent), and the lowest wavelength was calculated for the diclofenac in the gas phase [28].

\section{NMR spectrum analysis}

Molecular structure of the pethidine and diclofenac drugs were investigated using the B3PW91 method with DGDZVP basis set. The theoretical spectra were computed by Gaugeinvariant atomic orbital (GIAO) functional in combination with B3PW91 method and DGDZVP basis set in the gas and liquid phases.

The results depicted that ${ }^{13} \mathrm{C}-\mathrm{NMR}$ chemical shifts of pethidine were calculated by using B3PW91/DGDZVP, TMS B3LYP/6$311+\mathrm{G}(2 \mathrm{~d}, \mathrm{p}) \mathrm{GIAO}$ reference and gas phase in the range of 183-1 ppm, and at the presence of $\mathrm{H}_{2} \mathrm{O}, \mathrm{CH}_{3} \mathrm{OH}, \mathrm{C}_{2} \mathrm{H}_{5} \mathrm{OH}$, DMSO, $\mathrm{CH}_{3} \mathrm{CN}$ and $\mathrm{C}_{3} \mathrm{H}_{6} \mathrm{O}$ the solvents were calculated in the range of 185-1 ppm. Also, ${ }^{1} \mathrm{H}-\mathrm{NMR}$ chemical shifts were calculated using the B3PW91/DGDZVP and gas phase ranging from $8 \mathrm{ppm}$ to $0.4 \mathrm{ppm}$ at the presence of $\mathrm{H}_{2} \mathrm{O}, \mathrm{CH}_{3} \mathrm{OH}, \mathrm{C}_{2} \mathrm{H}_{5} \mathrm{OH}$, DMSO, $\mathrm{CH}_{3} \mathrm{CN}$, and $\mathrm{C}_{3} \mathrm{H}_{6} \mathrm{O}$ the solvents were calculated in the range of $8-0.30 \mathrm{ppm}$. Also, ${ }^{15} \mathrm{~N}-\mathrm{NMR}$ chemical shifts were calculated by using B3PW91/DGDZVP, NH3 B3LYP/6$311+G(2 d, p)$ GIAO reference and gas phase in the area $45.03 \mathrm{ppm}$ at the presence of $\mathrm{H}_{2} \mathrm{O}$, $\mathrm{CH}_{3} \mathrm{OH}, \mathrm{C}_{2} \mathrm{H}_{5} \mathrm{OH}$, DMSO, $\mathrm{CH}_{3} \mathrm{CN}$, and $\mathrm{C}_{3} \mathrm{H}_{6} \mathrm{O}$ the solvents were calculated at the range of 45-43 ppm. 
Table 2. Zero-point energy (ZPE), total energy $\left(E_{\mathrm{T}}, \mathrm{kcal} / \mathrm{mol}\right)$, Dipole Moment $(m)$ and polarizability $(\alpha, \mathrm{a}$. u) of pethidine and diclofenac drugs in various solvents

\begin{tabular}{ccccccccccccc}
\multicolumn{1}{c}{ Pethidine } & \multicolumn{1}{c}{ Diclofenac } \\
Media & $\mathrm{CH}_{3} \mathrm{CN}$ & $\mathrm{C}_{3} \mathrm{H}_{6} \mathrm{O}$ & DMSO & $\mathrm{C}_{2} \mathrm{H}_{5} \mathrm{OH}$ & $\mathrm{CH}_{3} \mathrm{OH}$ & $\mathrm{H}_{2} \mathrm{O}$ & $\mathrm{CH}_{3} \mathrm{CN}$ & $\mathrm{C}_{3} \mathrm{H}_{6} \mathrm{O}$ & $\mathrm{DMSO}$ & $\mathrm{C}_{2} \mathrm{H}_{5} \mathrm{OH}$ & $\mathrm{CH}_{3} \mathrm{OH}$ & $\mathrm{H}_{2} \mathrm{O}$ \\
$\mathrm{ZPE}$ & 0.344080 & 0.344097 & 0.344078 & 0.344092 & 0.344085 & 0.344072 & 0.225809 & 0.225836 & 0.225800 & 0.225825 & 0.225813 & 0.225788 \\
$E_{\mathrm{T}}$ & 226.672 & 226.680 & 226.669 & 226.677 & 226.673 & 226.665 & 152.105 & 152.117 & 152.101 & 152.112 & 152.107 & 152.096 \\
$\alpha$ & 202.395 & 200.465 & 203.035 & 201.248 & 202.144 & 203.877 & 223.609 & 221.487 & 224.310 & 222.349 & 223.334 & 225.231 \\
$m$ & 2.523 & 2.504 & 2.529 & 2.512 & 2.520 & 2.536 & 3.735 & 3.703 & 3.745 & 3.716 & 3.731 & 3.759 \\
\hline
\end{tabular}

Table 3. HOMO and LUMO energy value, ionization energy (I), electron affinity (A), chemical potential (P), global hardness ( $\eta$ ), global softness (S), electrophilicity index $(\omega)$, wavelength absorption $\left(\lambda_{\max }\right)$ and electro negativity $(\chi)$ of pethidine and diclofenac drugs in the gas phase and various solvents

\begin{tabular}{|c|c|c|c|c|c|c|c|c|c|c|c|}
\hline \multicolumn{12}{|c|}{ Pethidine } \\
\hline Media & $E(\mathrm{HOMO})$ & $E$ (LUMO) & $\Delta E$ & I & A & $\eta$ & $\mathrm{S}$ & $\chi$ & $\mathrm{P}$ & $\omega$ & $\lambda_{\max }$ \\
\hline $\mathrm{H}_{2} \mathrm{O}$ & -0.34086 & 0.10938 & 0.45024 & 0.34086 & -0.10938 & 0.225 & 2.221 & 0.231 & -0.231 & 0.118 & 200.72 \\
\hline DMSO & -0.34071 & 0.10953 & 0.45024 & 0.34071 & -0.10953 & 0.225 & 2.221 & 0.231 & -0.231 & 0.118 & 200.86 \\
\hline $\mathrm{C}_{2} \mathrm{H}_{5} \mathrm{OH}$ & -0.34039 & 0.10986 & 0.45025 & 0.34039 & -0.10986 & 0.225 & 2.220 & 0.230 & -0.230 & 0.117 & 200.82 \\
\hline $\mathrm{CH}_{3} \mathrm{CN}$ & -0.34059 & 0.10965 & 0.45024 & 0.34059 & -0.10965 & 0.225 & 2.221 & 0.230 & -0.230 & 0.117 & 200.77 \\
\hline $\mathrm{C}_{3} \mathrm{H}_{6} \mathrm{O}$ & -0.34024 & 0.11000 & 0.45024 & 0.34024 & -0.11000 & 0.225 & 2.221 & 0.230 & -0.230 & 0.117 & 200.84 \\
\hline \multicolumn{12}{|c|}{ Diclofenac } \\
\hline $\mathrm{H}_{2} \mathrm{O}$ & -0.30443 & 0.09401 & 0.39844 & 0.30443 & -0.09401 & 0.199 & 2.509 & 0.210 & -0.210 & 0.110 & 219.47 \\
\hline DMSO & -0.30435 & 0.09402 & 0.39837 & 0.30435 & -0.09402 & 0.199 & 2.510 & 0.210 & -0.210 & 0.110 & 220.24 \\
\hline $\mathrm{CH}_{3} \mathrm{OH}$ & -0.30427 & 0.09403 & 0.39830 & 0.30427 & -0.09403 & 0.199 & 2.510 & 0.210 & -0.210 & 0.110 & 219.47 \\
\hline $\mathrm{C}_{2} \mathrm{H}_{5} \mathrm{OH}$ & -0.30418 & 0.09404 & 0.39822 & 0.30418 & -0.09404 & 0.199 & 2.511 & 0.210 & -0.210 & 0.110 & 219.80 \\
\hline $\mathrm{CH}_{3} \mathrm{CN}$ & -0.30429 & 0.09403 & 0.39832 & 0.30429 & -0.09403 & 0.199 & 2.510 & 0.210 & -0.210 & 0.110 & 219.61 \\
\hline $\mathrm{C}_{3} \mathrm{H}_{6} \mathrm{O}$ & -0.30411 & 0.09405 & 0.39816 & 0.30411 & -0.09405 & 0.199 & 2.511 & 0.210 & -0.210 & 0.110 & 219.80 \\
\hline
\end{tabular}

HOMO $\rightarrow$ LUMO for pethidine $(67 \rightarrow 68)$ and HOMO $\rightarrow$ LUMO for diclofenac $(66 \rightarrow 67)$ 
Figure 3. The UV-vis absorption spectrum of pethidine and diclofenac drugs in the gas phase calculated by CISDGDZVP

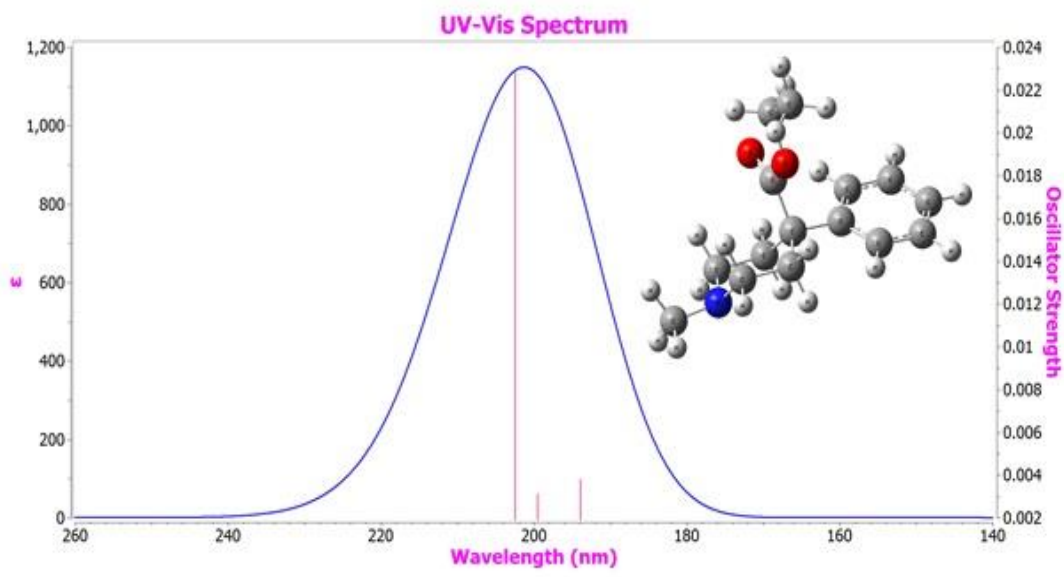

Pethidine

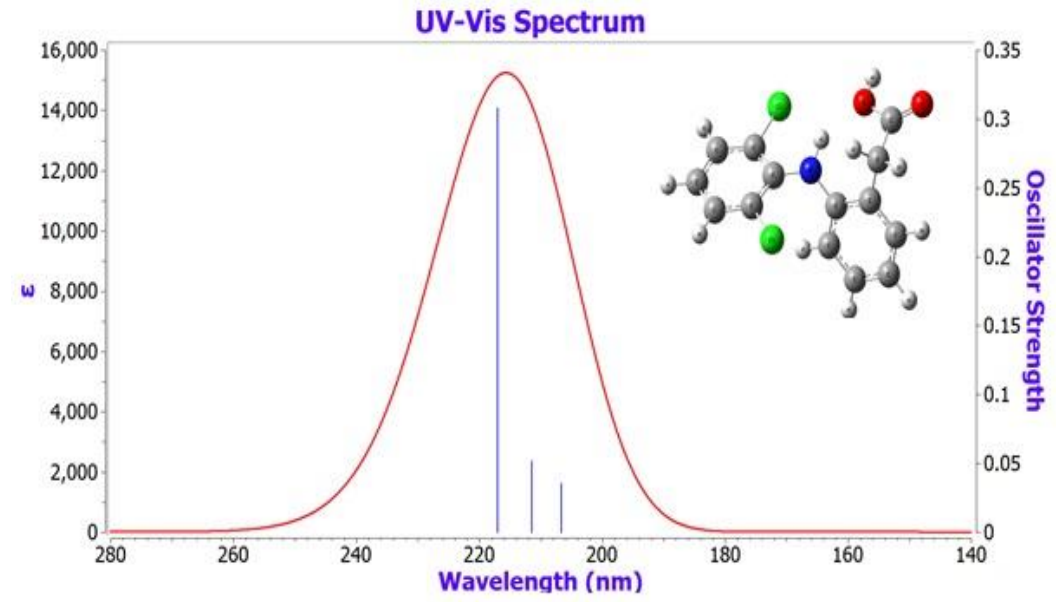

Diclofenac

The ${ }^{13} \mathrm{C}$-NMR chemical shifts of diclofenac were calculated using the B3PW91/DGDZVP, TMS B3LYP/6-311+G(2d,p) GIAO reference and gas phase in the range of 171-31 ppm, and at the presence of $\mathrm{H}_{2} \mathrm{O}, \mathrm{CH}_{3} \mathrm{OH}, \mathrm{C}_{2} \mathrm{H}_{5} \mathrm{OH}$, DMSO, $\mathrm{CH}_{3} \mathrm{CN}$ and $\mathrm{C}_{3} \mathrm{H}_{6} \mathrm{O}$ solvents were calculated in the range of 174-31 ppm. Also, $1 \mathrm{H}-\mathrm{NMR}$ chemical shifts were calculated by using B3PW91/DGDZVP and gas phase in the range of 8-3 ppm, and in the presence of $\mathrm{H}_{2} \mathrm{O}$, $\mathrm{CH}_{3} \mathrm{OH}, \mathrm{C}_{2} \mathrm{H}_{5} \mathrm{OH}$, DMSO, $\mathrm{CH}_{3} \mathrm{CN}$ and $\mathrm{C}_{3} \mathrm{H}_{6} \mathrm{O}$ solvents were calculated at the range of 8-2 ppm. Also, ${ }^{15} \mathrm{~N}-\mathrm{NMR}$ chemical shifts were calculated using B3PW91/DGDZVP, NH3 B3LYP/6-311+G(2d,p) GIAO reference and gas phase in the area $89.86 \mathrm{ppm}$, and in the presence of $\mathrm{H}_{2} \mathrm{O}, \mathrm{CH}_{3} \mathrm{OH}, \mathrm{C}_{2} \mathrm{H}_{5} \mathrm{OH}$, DMSO, $\mathrm{CH}_{3} \mathrm{CN}$ and $\mathrm{C}_{3} \mathrm{H}_{6} \mathrm{O}$ solvents were calculated in the range of 89-90 ppm [29].
Computational spectral data using B3PW91/DGDZVP

Pethidine: ${ }^{1} \mathrm{H}$ NMR (gas phase, $\delta$ ppm): $7.20(\mathrm{~d}, 1 \mathrm{H}), 7.01(\mathrm{~s}, 1 \mathrm{H}), 6.97(\mathrm{~s}, 1 \mathrm{H}), 6.96(\mathrm{~s}$, $1 \mathrm{H}), 6.93(\mathrm{~s}, 1 \mathrm{H}), 3.46(\mathrm{q}, 2 \mathrm{H}), 1.79(\mathrm{t}, 2 \mathrm{H}), 1.61$ $(\mathrm{t}, 2 \mathrm{H}), 1.55(\mathrm{t}, 2 \mathrm{H}), 1.72(\mathrm{~s}, 1 \mathrm{H}), 0.93(\mathrm{t}, 1 \mathrm{H})$, $0.44(\mathrm{t}, 3 \mathrm{H}), 0.36(\mathrm{t}, 1 \mathrm{H}), 0.04(\mathrm{t}, 1 \mathrm{H}) ;{ }^{13} \mathrm{C}$ NMR ( $\delta \mathrm{ppm}): 183.70,135.56,120.83,120.15$, 119.85, 117.71, 116.34, 55.39, 42.43, 42.31, 42.00, 34.48, 27.93, 23.85, 1.39; ${ }^{15} \mathrm{~N}$ NMR: 45.03; Diclofenac: ${ }^{1} \mathrm{H}$ NMR (gas phase, $\delta$ ppm): $7.15(\mathrm{~s}, 1 \mathrm{H}), 6.96(\mathrm{~d}, 1 \mathrm{H}), 6.82(\mathrm{t}, 1 \mathrm{H})$, $6.67(\mathrm{~d}, 1 \mathrm{H}), 5.95(\mathrm{~d}, 1 \mathrm{H}), 5.68(\mathrm{~s}, 1 \mathrm{H}), 3.07(\mathrm{~s}$, $1 \mathrm{H}) ;{ }^{13} \mathrm{C}$ NMR $(\delta \mathrm{ppm}): 171.70,132.18,131.33$, $131.11,128.60,122.96,122.70,120.92$, 120.56, 114.12, 113.75, 113.62, 110.29, 31.32; 15N NMR: 90.49. 
Pethidine: ${ }^{1} \mathrm{H}$ NMR $\left(\mathrm{H}_{2} \mathrm{O}, \delta \mathrm{ppm}\right): 7.29(\mathrm{~d}$, 1H), $7.24(\mathrm{~s}, 1 \mathrm{H}), 7.19(\mathrm{~s}, 1 \mathrm{H}), 7.18(\mathrm{~s}, 1 \mathrm{H}), 7.14$ $(\mathrm{s}, 1 \mathrm{H}), 3.60(\mathrm{q}, 2 \mathrm{H}), 1.82(\mathrm{t}, 2 \mathrm{H}), 1.68(\mathrm{t}, 2 \mathrm{H})$, $1.50(\mathrm{t}, 2 \mathrm{H}), 1.43(\mathrm{~s}, 1 \mathrm{H}), 1.36(\mathrm{~s}, 1 \mathrm{H}), 1.24(\mathrm{t}$, $2 \mathrm{H}), 0.87(\mathrm{t}, 1 \mathrm{H}), 0.48(\mathrm{t}, 1 \mathrm{H}), 0.31(\mathrm{t}, 1 \mathrm{H}), 0.30$ $(\mathrm{t}, 1 \mathrm{H}) ;{ }^{13} \mathrm{C}$ NMR $(\delta \mathrm{ppm}): 185.40,135.50$, $120.54,120.41,120.37,118.38,116.51,56.69$, 42.52, 41.83, 41.63, 33.82, 27.97, 24.03, 1.00; ${ }^{15} \mathrm{~N}$ NMR: 43.97; Diclofenac: ${ }^{1} \mathrm{H}$ NMR $\left(\mathrm{H}_{2} \mathrm{O}, \delta\right.$ ppm): $7.22(\mathrm{~s}, 1 \mathrm{H}), 7.19(\mathrm{~d}, 1 \mathrm{H}), 7.05(\mathrm{t}, 1 \mathrm{H})$, $7.04(\mathrm{~d}, 1 \mathrm{H}), 7.00(\mathrm{~d}, 1 \mathrm{H}), 6.92(\mathrm{~d}, 1 \mathrm{H}), 6.75(\mathrm{~d}$, $1 \mathrm{H}), 6.47(\mathrm{~s}, 1 \mathrm{H}), 6.14(\mathrm{~d}, 1 \mathrm{H}), 3.20(\mathrm{~s}, 1 \mathrm{H})$, $2.63(\mathrm{~s}, 1 \mathrm{H}) ;{ }^{13} \mathrm{C}$ NMR $(\delta \mathrm{ppm}): 174.78,132.47$, $130.93,129.78,127.84,123.55,123.40$, $122.33,120.51,115.43,114.32,114.02$, 110.01, 31.46; ${ }^{15}$ N NMR: 89.86.

Pethidine: ${ }^{1} \mathrm{H}$ NMR $\left(\mathrm{CH}_{3} \mathrm{OH}, \delta \mathrm{ppm}\right): 7.28$ (d, 1H), $7.23(\mathrm{~s}, 1 \mathrm{H}), 7.19(\mathrm{~s}, 1 \mathrm{H}), 7.18(\mathrm{~s}, 1 \mathrm{H})$, $7.13(\mathrm{~s}, 1 \mathrm{H}), 3.60(\mathrm{q}, 1 \mathrm{H}), 3.24(\mathrm{q}, 1 \mathrm{H}), 1.82(\mathrm{t}$, 1H), $1.76(\mathrm{~d}, 1 \mathrm{H}), 1.68(\mathrm{~d}, 1 \mathrm{H}), 1.50(\mathrm{t}, 2 \mathrm{H})$, $1.42(\mathrm{~s}, 1 \mathrm{H}), 1.35(\mathrm{~s}, 1 \mathrm{H}), 1.24(\mathrm{t}, 2 \mathrm{H}), 0.87(\mathrm{t}$, $1 \mathrm{H}), 0.48(\mathrm{t}, 1 \mathrm{H}), 0.30(\mathrm{t}, 2 \mathrm{H}) ;{ }^{13} \mathrm{C}$ NMR $(\delta$ ppm): 185.35, 135.51, 120.51, 120.39, 120.38, 118.35, 116.51, 56.65, 42.52, 41.86, 41.65, 33.84, 27.96, 24.01, 1.01; ${ }^{15} \mathrm{~N}$ NMR: 44.01; Diclofenac: ${ }^{1} \mathrm{H}$ NMR $\left(\mathrm{CH}_{3} \mathrm{OH}, \delta\right.$ ppm): $7.22(\mathrm{~s}$, $1 \mathrm{H}), 7.18(\mathrm{~d}, 1 \mathrm{H}), 7.04(\mathrm{t}, 1 \mathrm{H}), 7.03(\mathrm{~d}, 1 \mathrm{H})$, $7.00(\mathrm{~d}, 1 \mathrm{H}), 6.91(\mathrm{~d}, 1 \mathrm{H}), 6.74(\mathrm{~d}, 1 \mathrm{H}), 6.45(\mathrm{~s}$, $1 \mathrm{H}), 6.13(\mathrm{~d}, 1 \mathrm{H}), 3.20(\mathrm{~s}, 1 \mathrm{H}), 2.62(\mathrm{~s}, 1 \mathrm{H}) ;{ }^{13} \mathrm{C}$ NMR ( $\delta$ ppm): 174.71, 132.46, 130.94, 129.82, $127.87,123.52,123.39,122.29,120.51$, $115.37,114.30,114.02,110.02,31.46 ;{ }^{15} \mathrm{~N}$ NMR: 89.89.

Pethidine: ${ }^{1} \mathrm{H}$ NMR $\left(\mathrm{C}_{2} \mathrm{H}_{5} \mathrm{OH}, \delta\right.$ ppm $): 7.28$ (d, 1H), 7.22 (s, 1H), $7.18(\mathrm{~s}, 2 \mathrm{H}), 7.13(\mathrm{~s}, 1 \mathrm{H})$, $3.59(\mathrm{q}, 1 \mathrm{H}), 3.24(\mathrm{q}, 1 \mathrm{H}), 1.82(\mathrm{t}, 1 \mathrm{H}), 1.75(\mathrm{~d}$, $1 \mathrm{H}), 1.68(\mathrm{~d}, 1 \mathrm{H}), 1.50(\mathrm{t}, 2 \mathrm{H}), 1.41(\mathrm{~s}, 1 \mathrm{H})$, $1.35(\mathrm{~s}, 1 \mathrm{H}), 1.25(\mathrm{t}, 2 \mathrm{H}), 0.88(\mathrm{t}, 1 \mathrm{H}), 0.48(\mathrm{t}$, $1 \mathrm{H}), 0.30(\mathrm{t}, 2 \mathrm{H}) ;{ }^{13} \mathrm{C}$ NMR $(\delta \mathrm{ppm}): 185.33$, $135.51,120.50,120.39,118.33,116.51,56.63$, 42.51, 41.88, 41.66, 33.86, 27.96, 24.01, 1.02; ${ }^{15} \mathrm{~N}$ NMR: 44.03; Diclofenac: ${ }^{1} \mathrm{H} \quad \mathrm{NMR}$ $\left(\mathrm{C}_{2} \mathrm{H}_{5} \mathrm{OH}, \delta \mathrm{ppm}\right): 7.21(\mathrm{~s}, 1 \mathrm{H}), 7.18(\mathrm{~d}, 1 \mathrm{H})$, $7.04(\mathrm{t}, 1 \mathrm{H}), 7.03(\mathrm{~d}, 1 \mathrm{H}), 6.99(\mathrm{~d}, 1 \mathrm{H}), 6.91(\mathrm{~d}$, $1 \mathrm{H}), 6.73(\mathrm{~d}, 1 \mathrm{H}), 6.44(\mathrm{~s}, 1 \mathrm{H}), 6.13(\mathrm{~d}, 1 \mathrm{H})$, 3.19 (s, 1H), $2.62(\mathrm{~s}, 1 \mathrm{H}) ;{ }^{13} \mathrm{C}$ NMR $(\delta \mathrm{ppm})$ :
174.67, 132.46, 130.94, 129.84, 127.88, $123.51,123.38,122.26,120.51,115.33$, 114.29, 114.02, 110.02, 31.45; $15 \mathrm{~N}$ NMR: 89.90.

Pethidine: ${ }^{1} \mathrm{H}$ NMR (DMSO, $\delta$ ppm): 7.29 (d, 1H), 7.23 (s, 1H), 7.19 (s, 2H), $7.18(\mathrm{~s}, 1 \mathrm{H})$, $3.60(\mathrm{q}, 1 \mathrm{H}), 3.24(\mathrm{q}, 1 \mathrm{H}), 1.82(\mathrm{t}, 1 \mathrm{H}), 1.76(\mathrm{~d}$, $1 \mathrm{H}), 1.68(\mathrm{~d}, 1 \mathrm{H}), 1.50(\mathrm{t}, 2 \mathrm{H}), 1.42(\mathrm{~s}, 1 \mathrm{H})$, $1.35(\mathrm{~s}, 1 \mathrm{H}), 1.24(\mathrm{t}, 2 \mathrm{H}), 0.87(\mathrm{t}, 1 \mathrm{H}), 0.48(\mathrm{t}$, $1 \mathrm{H}), 0.31(\mathrm{t}, 2 \mathrm{H}) ;{ }^{13} \mathrm{C}$ NMR $(\delta \mathrm{ppm}): 185.38$, $135.50,120.53,120.40,120.38,118.36$, 116.51, 56.67, 42.52, 41.85, 41.64, 33.83, 27.97, 24.02, 1.01; ${ }^{15} \mathrm{~N}$ NMR: 43.99; Diclofenac: ${ }^{1} \mathrm{H}$ NMR (DMSO, $\delta$ ppm): $7.22(\mathrm{~s}$, 1H), $7.18(\mathrm{~d}, 1 \mathrm{H}), 7.05(\mathrm{t}, 1 \mathrm{H}), 7.04(\mathrm{~d}, 1 \mathrm{H})$, $7.00(\mathrm{~d}, 1 \mathrm{H}), 6.91(\mathrm{~d}, 1 \mathrm{H}), 6.74(\mathrm{~d}, 1 \mathrm{H}), 6.46(\mathrm{~s}$, $1 \mathrm{H}), 6.13(\mathrm{~d}, 1 \mathrm{H}), 3.20(\mathrm{~s}, 1 \mathrm{H}), 2.63(\mathrm{~s}, 1 \mathrm{H}) ;{ }^{13} \mathrm{C}$ NMR ( $\delta$ ppm): 174.75, 132.47, 130.93, 129.80, $127.85,123.54,123.40,122.31,120.51$, $115.40,114.31,114.02,110.01,31.46 ;{ }^{15} \mathrm{~N}$ NMR: 89.88.

Pethidine: ${ }^{1} \mathrm{H}$ NMR $\left(\mathrm{CH}_{3} \mathrm{CN}, \delta\right.$ ppm): 7.28 (d, 1H), 7.23 (s, 1H), 7.19 (s, 2H), $7.13(\mathrm{~s}, 1 \mathrm{H})$, $3.60(\mathrm{q}, 1 \mathrm{H}), 3.24(\mathrm{q}, 1 \mathrm{H}), 1.82(\mathrm{t}, 1 \mathrm{H}), 1.76(\mathrm{~d}$, $1 \mathrm{H}), 1.64(\mathrm{~d}, 1 \mathrm{H}), 1.51(\mathrm{t}, 2 \mathrm{H}), 1.42(\mathrm{~s}, 1 \mathrm{H})$, $1.35(\mathrm{~s}, 1 \mathrm{H}), 1.24(\mathrm{t}, 2 \mathrm{H}), 0.87(\mathrm{t}, 1 \mathrm{H}), 0.48(\mathrm{t}$, $1 \mathrm{H}), 0.30(\mathrm{t}, 2 \mathrm{H}) ;{ }^{13} \mathrm{C}$ NMR $(\delta \mathrm{ppm}): 185.36$, $135.50,120.52,120.39,120.38,118.35$, 116.51, 56.66, 42.52, 41.86, 41.65, 33.84, 27.96, 24.02, $1.01 ;{ }^{15} \mathrm{~N} \quad \mathrm{NMR}: 44.00$; Diclofenac: ${ }^{1} \mathrm{H}$ NMR $\left(\mathrm{CH}_{3} \mathrm{CN}, \delta \mathrm{ppm}\right): 7.22(\mathrm{~s}$, $1 \mathrm{H}), 7.18(\mathrm{~d}, 1 \mathrm{H}), 7.04(\mathrm{t}, 1 \mathrm{H}), 7.03(\mathrm{~d}, 1 \mathrm{H})$, $7.00(\mathrm{~d}, 1 \mathrm{H}), 6.91(\mathrm{~d}, 1 \mathrm{H}), 6.74(\mathrm{~d}, 1 \mathrm{H}), 6.45(\mathrm{~s}$, $1 \mathrm{H}), 6.13(\mathrm{~d}, 1 \mathrm{H}), 3.20(\mathrm{~s}, 1 \mathrm{H}), 2.62(\mathrm{~s}, 1 \mathrm{H}) ;{ }^{13} \mathrm{C}$ NMR ( $\delta$ ppm): 174.72, 132.46, 130.94, 129.81, $127.86,123.53,123.39,122.29,120.51$, $115.37,114.30,114.02,110.01,31.46 ;{ }^{15} \mathrm{~N}$ NMR: 89.89.

Pethidine: ${ }^{1} \mathrm{H}$ NMR $\left(\mathrm{C}_{3} \mathrm{H}_{6} \mathrm{O}, \delta\right.$ ppm $): 7.27$ (d, 1H), $7.22(\mathrm{~s}, 1 \mathrm{H}), 7.18(\mathrm{~s}, 2 \mathrm{H}), 7.13(\mathrm{~s}, 1 \mathrm{H})$, $3.59(\mathrm{q}, 1 \mathrm{H}), 3.24(\mathrm{q}, 1 \mathrm{H}), 1.82(\mathrm{t}, 1 \mathrm{H}), 1.75(\mathrm{~d}$, $1 \mathrm{H}), 1.68(\mathrm{~d}, 1 \mathrm{H}), 1.50(\mathrm{t}, 2 \mathrm{H}), 1.41(\mathrm{~s}, 1 \mathrm{H})$, $1.34(\mathrm{~s}, 1 \mathrm{H}), 1.25(\mathrm{t}, 2 \mathrm{H}), 0.88(\mathrm{t}, 1 \mathrm{H}), 0.48(\mathrm{t}$, $1 \mathrm{H}), 0.31(\mathrm{t}, 2 \mathrm{H}) ;{ }^{13} \mathrm{C}$ NMR $(\delta \mathrm{ppm}): 185.35$, $135.51,120.49,120.40,120.38,118.32$, 116.51, 56.61, 42.51, 41.89, 41.67, 33.87, 
27.96, 24.00, 1.02; ${ }^{15 \mathrm{~N}}$ NMR: 44.04; Diclofenac: ${ }^{1} \mathrm{H}$ NMR $\left(\mathrm{C}_{3} \mathrm{H}_{6} \mathrm{O}, \delta \mathrm{ppm}\right): 7.21(\mathrm{~s}$, $1 \mathrm{H}), 7.17(\mathrm{~d}, 1 \mathrm{H}), 7.03(\mathrm{t}, 1 \mathrm{H}), 7.02(\mathrm{~d}, 1 \mathrm{H})$, $6.99(\mathrm{~d}, 1 \mathrm{H}), 6.90(\mathrm{~d}, 1 \mathrm{H}), 6.72(\mathrm{~d}, 1 \mathrm{H}), 6.43(\mathrm{~s}$, $1 \mathrm{H}), 6.12(\mathrm{~d}, 1 \mathrm{H}), 3.19(\mathrm{~s}, 1 \mathrm{H}), 2.62(\mathrm{~s}, 1 \mathrm{H}) ;{ }^{13} \mathrm{C}$ NMR ( $\delta$ ppm): $174.64,132.45,130.94,129.86$, $127.90,123.50,123.38,122.24,120.51$, $115.31,114.28,114.02,110.02,31.45 ;{ }^{15} \mathrm{~N}$ NMR: 89.91.

\section{Frontier molecular orbital (FMO) analysis}

Frontier molecular orbitals, both the highest occupied molecular orbital (HOMO) and lowest unoccupied molecular orbital (LUMO) and their energy gap, are very useful parameters for quantum chemistry. These energy gaps showed that, the titled molecules are stable. A molecule having a small frontier orbital gap is more polarizable and is generally associated with a high chemical reactivity and low kinetic stability. In this study, ionization energy, electron affinity, chemical potential, global hardness, global softness, electrophilicity index, electro negativity and $\Delta \mathrm{E}_{\text {номо - Luмо for pethidine and }}$ diclofenac drugs were calculated using the B3PW91/DGDZVP in the gas phase and various solvents including, $\mathrm{H}_{2} \mathrm{O}, \mathrm{CH}_{3} \mathrm{OH}$, $\mathrm{C}_{2} \mathrm{H}_{5} \mathrm{OH}$, DMSO, $\mathrm{CH}_{3} \mathrm{CN}$, and $\mathrm{C}_{3} \mathrm{H}_{6} \mathrm{O}$ (Figure 4).

The following equations were used to calculate the ionization energy (I), electron affinity (A), dipole moment $(\mu)$, chemical potential $(\mathrm{P})$, global hardness $(\eta)$, global softness (S), electrophilicity index $(\omega)$ and electro negativity $(\chi)$. The results are summarized in Table 3.

$$
\begin{aligned}
& \eta=\frac{1}{2}(\mathrm{I}-\mathrm{A}) \\
& \mathrm{S}=\frac{1}{2 \eta} \\
& \mu=-\frac{1}{2}(\mathrm{I}+\mathrm{A}) \\
& \omega=\frac{\mu^{2}}{2 \eta} \\
& \mathrm{X}=\frac{1}{2}(\mathrm{I}+\mathrm{A})
\end{aligned}
$$

$\mathrm{I}=\left(-E_{\text {Номо }}\right)$

$\mathrm{A}=\left(-E_{L U M O}\right)$

The highest energy gap was calculated for pethidine equal to $0.45059 \mathrm{eV}$ in the gas phase, and it was calculated for diclofenac equal to $0.39844 \mathrm{eV}$ at the presence of $\mathrm{H}_{2} \mathrm{O}$ solvent. This suggests that the diclofenac has the highest stability at the presence of solvent. However, pethidine revealed the highest stability in the gas phase. Also, for pethidine, HOMO $\rightarrow$ LUMO was electron transfer from electron levels of $67 \rightarrow 68$ and for diclofenac, transfer was from electron levels of $66 \rightarrow 67$. The highest electro negativity was calculated for the pethidine by using B3PW91/DGDZVP equal to $0.231 \mathrm{eV}$ in the presence of $\mathrm{H}_{2} \mathrm{O}$ and DMSO solvents, and it was calculated for diclofenac equal to $0.210 \mathrm{eV}$ at the presence of solvents such as $\mathrm{H}_{2} \mathrm{O}, \mathrm{CH}_{3} \mathrm{OH}, \mathrm{C}_{2} \mathrm{H}_{5} \mathrm{OH}$, DMSO, $\mathrm{CH}_{3} \mathrm{CN}$, and $\mathrm{C}_{3} \mathrm{H}_{6} \mathrm{O}$. Also, pethidine revealed the highest electrophilicity index $(0.118 \mathrm{eV})$ at the presence of $\mathrm{H}_{2} \mathrm{O}$ and DMSO solvents, and it was calculated for diclofenac drug equal to $0.110 \mathrm{eV}$ in the gas phase and various solvents [30].

\section{Natural population analysis}

The natural bond orbitals calculations were performed using the Gaussian $09 \mathrm{~W}$ package using the (DFT/B3PW91) method and DGDZVP level of theory. The second order Fock matrix, natural population analysis, hybridization, and natural electronic configuration were carried out to evaluate the donor-acceptor interactions in the NBO analysis [31].

In this study, Mulliken and natural atomic charges between the atoms of pethidine and diclofenac drugs were calculated using the B3PW91 method with DGDZVP basis set (Figure 5). Natural charges revealed that the six carbon atoms in the benzene ring, $\mathbf{0}_{\mathbf{9}}, \mathbf{0}_{\mathbf{1 0}}$, and $\mathbf{N}_{\mathbf{1 5}}$ had partially negative charges in the optimized geometry of pethidine. Also, the largest positive charge is located on $\mathbf{C}_{\mathbf{8}}$ atom, 
and the largest negative charge was located on $\mathbf{O}_{\mathbf{1 0}}$ atom. Natural charges revealed that the five carbon atoms $\left(\mathrm{C}_{1}, \mathrm{C}_{2}, \mathrm{C}_{3}, \mathrm{C}_{4}\right.$ and $\left.\mathrm{C}_{5}\right)$ in the

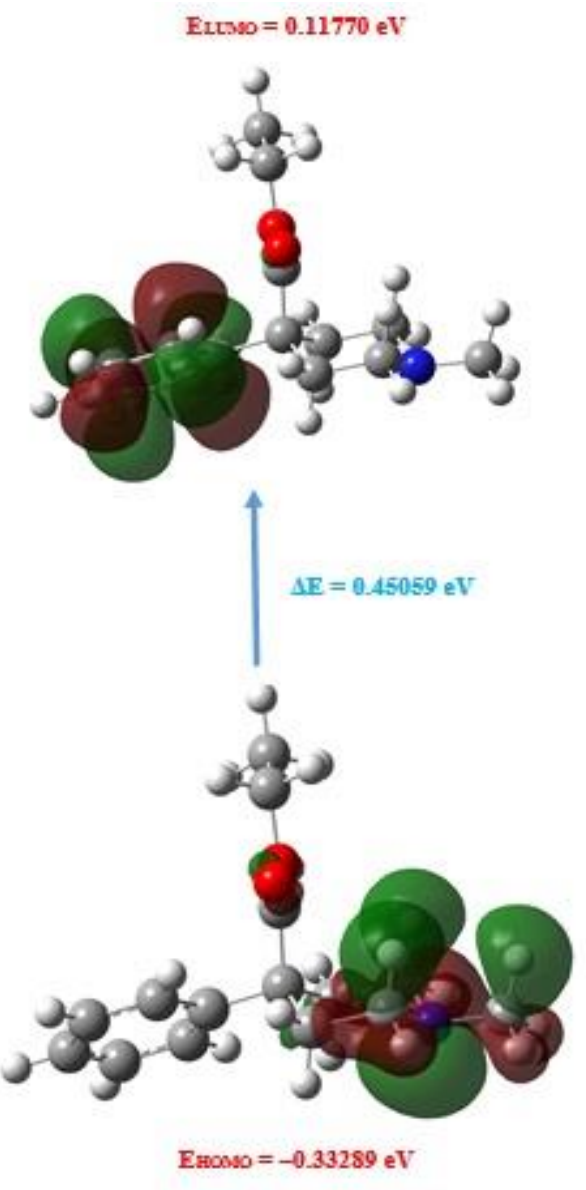

Pethidine benzene ring, $\mathbf{O}_{\mathbf{9}}, \mathbf{O}_{\mathbf{1 8}}, \mathbf{C l}_{\mathbf{1 4}}, \mathbf{C l}_{\mathbf{1 5}}$, and $\mathbf{N 7}$ had partially negative charges in the optimized geometry of diclofenac.

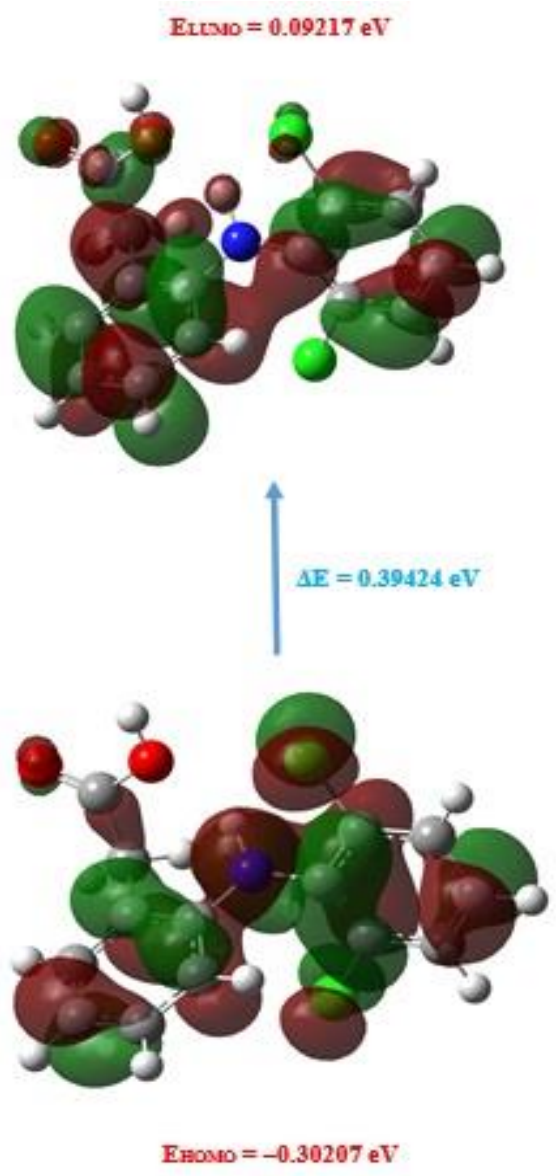

Diclofenac

Figure 4. Frontier molecular orbitals of pethidine and diclofenac drugs in the gas phase calculated by B3PW91/DGDZVP

The largest positive charge was found on $\mathbf{C}_{17}$ atom, and the largest negative charge was located on the $\mathbf{O}_{18}$ atom. The computed 3D plot of molecular electrostatic potential (MEP) and total density in the gas phase for pethidine and diclofenac drugs is depicted in Figure 6, which is based on the electron density at different points on the molecule. As seen in Figure 6, the regions surrounding the oxygen atoms are strongly negative, while the $\mathrm{H}$ atoms in the ring and methyl group are positive; among them the $\mathrm{H}$ atoms in the ring are more positive compared with that of the methyl groups.

\section{Natural bond orbital (NBO) analysis}

Natural bond orbital analysis is a useful method for determining the electric charge of atoms and natural electron configuration includes orbitals s, $\mathbf{p}, \mathbf{d}$, and $\mathbf{f}$ [32]. Pethidine has 39 atoms including $\mathrm{C}, \mathrm{H}, \mathrm{N}$, and $\mathrm{O}$. According to the NBO results, $\mathbf{O}_{9}$ and $\mathbf{O}_{10}$ atoms with $\mathbf{C}_{\mathbf{8}}$ make the bond, which the electron configuration of $\mathbf{0}_{\mathbf{1 0}}$ is: [core] $2 s^{1.73} 2 p^{5.00} 3 p^{0.01}$. Thus, 1.99986 core electrons, 6.728 valence electrons (on $2 s$ and $2 p$ atomic orbitals (AO)), and 0.008 Rydberg electrons (mainly on $3 p$ ) give a total of 8.736 electrons. Natural electron configuration of $\quad \mathbf{C}_{\mathbf{8}}$ is: core $] 2 s^{0.79} 2 p^{2.15} 3 s^{0.01} 3 p^{0.03}$. 
Figure 5. (A) Mulliken charge and natural atomic charge distribution of pethidine and drug and (B) Mulliken charge and natural atomic charge distribution of diclofenac drug by B3PW91/DGDZVP
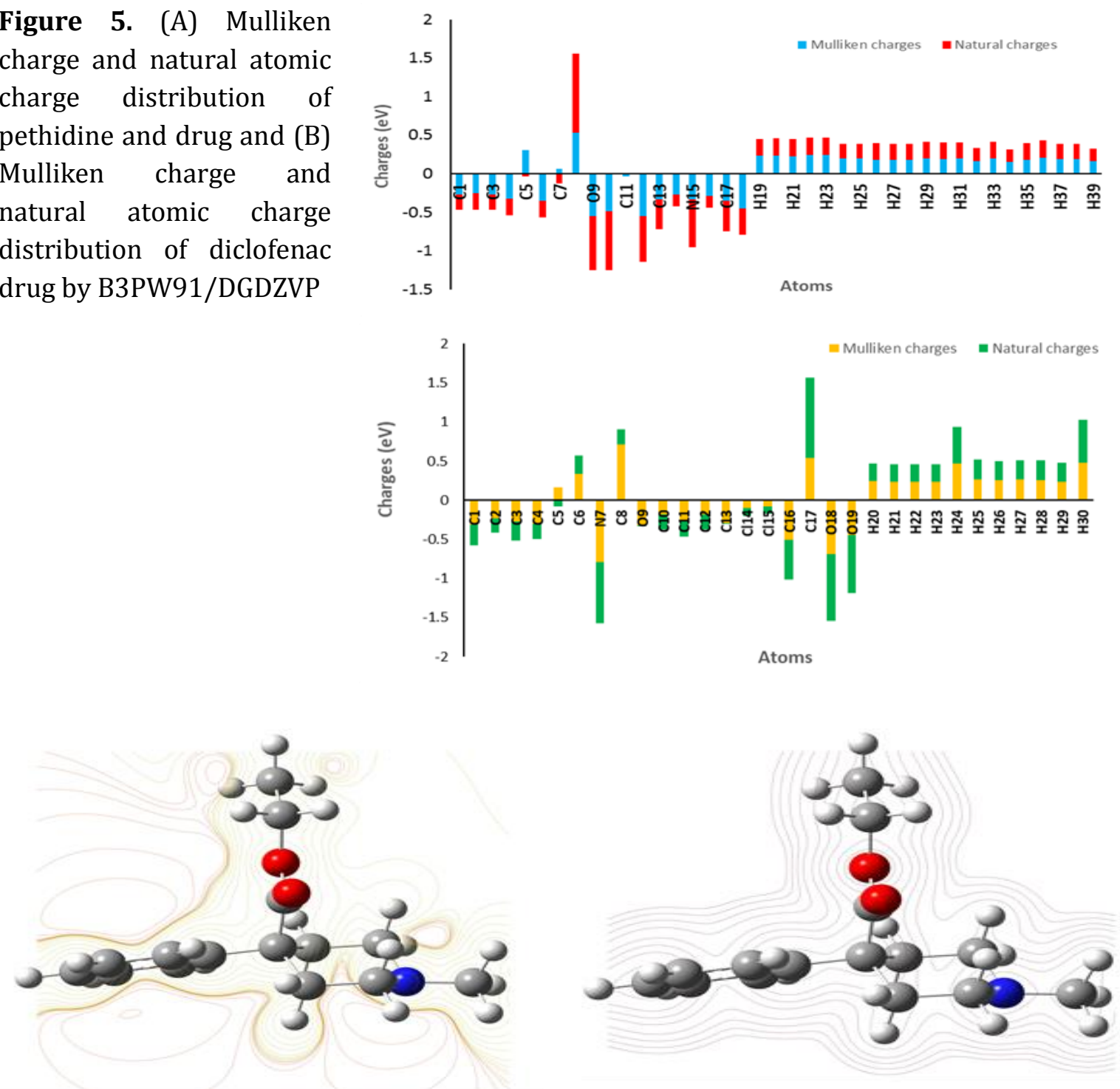

\section{Pethidine}

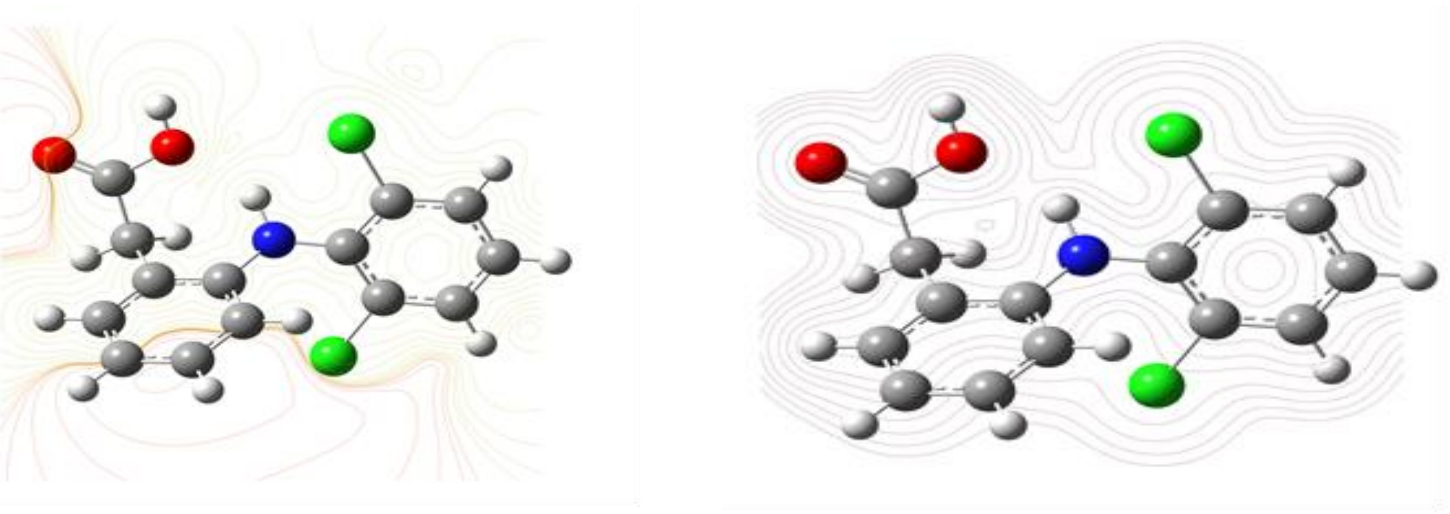

\section{Diclofenac}

Figure 6. Molecular electrostatic potential (MESP) and total density in the gas phase of pethidine and diclofenac drugs

Thus, 1.99920 core electrons, 2.943 valence electrons (on $2 \mathrm{~s}$ and $2 \mathrm{p}$ atomic orbitals (AO)), and 0.046 Rydberg electrons (mainly on 3 s and $3 p$ ) give a total of 4.988 electrons (Table 4 ). 
diclofenac has 30 atoms including $\mathrm{C}, \mathrm{H}, \mathrm{N}, \mathrm{O}$ and $\mathrm{Cl}$ each of these atoms are different natural electron configuration. According to the NBO results, $\mathbf{C}_{\mathbf{8}}$ and $\mathbf{C}_{6}$ atoms with $\mathbf{N}_{7}$ makes the bond, that the electron configuration of $\mathbf{N}_{7}$ is: [core] $2 s^{1.25} 2 p^{4.50} 3 p^{0.02}$. Thus, 1.99940 core electrons, 5.750 valence electrons (on $2 s$ and $2 p$ atomic orbitals (AO)), and 0.022 Rydberg electrons (mainly on $3 \mathrm{p}$ ) give a total of 7.772 electrons. $\mathbf{C}_{\mathbf{9}}$ and $\mathbf{C}_{\mathbf{1 3}}$ atoms with $\mathbf{C l}_{\mathbf{1 4}}$ and $\mathbf{C l}_{\mathbf{1 5}}$ makes the bond, that the electron configuration of $\mathbf{C l}_{\mathbf{1 4}}$ is: [core] $3 s^{1.83} 3 p^{5.25}$. Thus, 10.00 core electrons, 7.087 valence electrons (on $2 s$ and $2 p$ atomic orbitals (AO)), and 0.002 Rydberg electrons give a total of 17.090 electrons.

Also, natural bond orbital analysis can show occupancy and hybridization between atoms. In this study, the type of hybridization between $\mathrm{C}-\mathrm{O}, \mathrm{C}-\mathrm{N}, \mathrm{C}=\mathrm{O}, \mathrm{C}=\mathrm{C}, \mathrm{C}-\mathrm{C}$ and $\mathrm{C}-\mathrm{Cl}$ bonds and the contribution of each of the orbitals were calculated in bonds (Table 5). In the molecular structure of pethidine, the $\pi\left(\mathrm{C}_{8}-\mathrm{O}_{10}\right)$ bond is formed from an $s p^{2.04}$ hybrid on $\mathbf{C}$ (which is a mixture of $32.93 \% s$ and $67.07 \% p \mathrm{AO}$ ) and $s p^{1.53}$ hybrid on $\mathbf{O}$ (which is a mixture of $39.52 \% s$ and $60.48 \% p \mathrm{AO}$ ), including square of polarization co-efficient $(32.72 \% \mathrm{C}$ and $67.28 \%$ $0)$. In the molecular structure of diclofenac, the $\sigma\left(\mathrm{C}_{9}-\mathrm{Cl}_{14}\right)$ bond is formed from an $s p^{3.84}$ hybrid on $\mathbf{C}$ (which is a mixture of $20.65 \% s$ and $79.35 \% p \mathrm{AO}$ ) and $s p^{4.26}$ hybrid on $\mathbf{C l}$ (which is a mixture of $19.02 \% s$ and $80.98 \% p$ AO), including square of polarization co-efficient ( $42.18 \% \mathrm{C}$ and $57.82 \% \mathrm{Cl}$ ).

The analysis of the various donors and acceptors indicate that there are only three types of donors $\sigma, \pi \& \mathrm{LP}$, and two types of acceptors $\sigma^{*}$ and $\pi^{*}$ [33]. The observation of excitation energy $E^{(2)}$ for different transitions among these donors and acceptors shows that the chances for the pethidine drug following transitions are highly probable; from $\mathrm{C}_{1}-\mathrm{C}_{2} \rightarrow$ $\mathrm{C}_{3}-\mathrm{C}_{4}\left(43.180 \mathrm{kj} / \mathrm{mol}, \pi \rightarrow \pi^{*}\right), \mathrm{C}_{13}-\mathrm{C}_{14} \rightarrow$ $\mathrm{N}_{15}-\mathrm{C}_{18}\left(5.070 \mathrm{kj} / \mathrm{mol}, \sigma \rightarrow \pi^{*}\right), \mathrm{O}_{9} \rightarrow \mathrm{C}_{8}-\mathrm{O}_{10}$ $\left(63.110 \mathrm{kj} / \mathrm{mol}\right.$, LP (2) $\left.\rightarrow \pi^{*}\right)$ and $\mathrm{O}_{10} \rightarrow \mathrm{C}_{8}-\mathrm{O}_{9}$ (37.510 kj/mol, LP (2) $\rightarrow \sigma^{*}$ ). For diclofenac drug following transitions are highly probable; from $\mathrm{C}_{3}-\mathrm{C}_{4} \rightarrow \mathrm{C}_{1}-\mathrm{C}_{2}\left(48.650 \mathrm{kj} / \mathrm{mol}, \pi \rightarrow \pi^{*}\right)$, $\mathrm{C}_{8}-\mathrm{C}_{9} \rightarrow \mathrm{C}_{10}-\mathrm{C}_{11}\left(49.370 \mathrm{kj} / \mathrm{mol}, \pi \rightarrow \pi^{*}\right), \mathrm{O}_{18} \rightarrow$ $\mathrm{C}_{17}-\mathrm{O}_{19}\left(50.74 \mathrm{kj} / \mathrm{mol}, \mathrm{LP}(2) \rightarrow \pi^{*}\right)$ and $\mathrm{O}_{18} \rightarrow$ $\mathrm{C}_{17}-\mathrm{O}_{18}\left(42.99 \mathrm{kj} / \mathrm{mol}, \mathrm{LP}(2) \rightarrow \pi^{*}\right.$ ) (Table 6).

Table 4. Summary of natural population analysis, natural electronic configuration of pethidine and diclofenac drugs by B3PW91/DGDZVP

\begin{tabular}{|c|c|c|c|c|c|c|}
\hline & \multirow[t]{2}{*}{ Atoms } & \multicolumn{4}{|c|}{ Natural population } & \multirow{2}{*}{$\begin{array}{l}\text { Natural electron } \\
\text { configuration }\end{array}$} \\
\hline & & Core & Valence & Rydberg & Total & \\
\hline \multirow[t]{6}{*}{ Pethidine } & $\mathrm{C}_{7}$ & 1.99914 & 4.090 & 0.030 & 6.120 & [core] $2 s^{0.93} 2 p^{3.16} 3 p^{0.03}$ \\
\hline & $\mathrm{C}_{8}$ & 1.99920 & 2.943 & 0.046 & 4.988 & [core] $2 s^{0.79} 2 p^{2.15} 3 s^{0.01} 3 p^{0.03}$ \\
\hline & $\mathbf{O}_{9}$ & 1.99981 & 6.687 & 0.011 & 8.699 & [core] $2 s^{1.65} 2 p^{5.03} 3 p^{0.01}$ \\
\hline & $\mathbf{O}_{10}$ & 1.99986 & 6.728 & 0.008 & 8.736 & [core] $2 s^{1.73} 2 p^{5.00} 3 p^{0.01}$ \\
\hline & $\mathrm{C}_{14}$ & 1.99934 & 4.140 & 0.019 & 6.160 & [core] $2 s^{1.00} 2 p^{3.14} 3 p^{0.02}$ \\
\hline & $\mathbf{N}_{15}$ & 1.99960 & 5.584 & 0.017 & 7.601 & {$\left[\right.$ core] $2 s^{1.31} 2 p^{4.27} 3 p^{0.02}$} \\
\hline \multicolumn{7}{|l|}{ Diclofenac } \\
\hline & $\mathrm{C}_{17}$ & 1.99946 & 2.958 & 0.032 & 4.990 & [core] $2 s^{0.80} 2 p^{2.16} 3 s^{0.01} 3 p^{0.02}$ \\
\hline & $\mathbf{O}_{18}$ & 1.99986 & 6.838 & 0.008 & 8.846 & [core] $2 s^{1.71} 2 p^{5.13} 3 p^{0.01}$ \\
\hline & $\mathbf{O}_{19}$ & 1.99988 & 6.683 & 0.009 & 8.692 & [core] $2 s^{1.73} 2 p^{4.95} 3 p^{0.01}$ \\
\hline & $\mathbf{N}_{7}$ & 1.99940 & 5.750 & 0.022 & 7.772 & [core] $2 s^{1.25} 2 p^{4.50} 3 p^{0.02}$ \\
\hline & $\mathrm{Cl}_{14}$ & 10.0000 & 7.087 & 0.002 & 17.090 & [core] $3 s^{1.83} 3 p^{5.25}$ \\
\hline & $\mathrm{Cl}_{15}$ & 10.0000 & 7.063 & 0.003 & 17.066 & [core] $3 s^{1.83} 3 p^{5.23}$ \\
\hline
\end{tabular}


Table 5. The NBO results for occupancies and hybridization of $\mathrm{C}-\mathrm{O}, \mathrm{C}-\mathrm{N}, \mathrm{C}=\mathrm{O}, \mathrm{C}=\mathrm{C}, \mathrm{C}-\mathrm{C}$ and $\mathrm{C}-\mathrm{Cl}$ bonds

\begin{tabular}{|c|c|c|c|c|c|c|c|c|}
\hline \multicolumn{2}{|c|}{ CAM-B3LYP/LanL2DZ } & \multicolumn{4}{|c|}{$[\mathrm{AO}] \%$} & \multirow{2}{*}{\multicolumn{2}{|c|}{ S.P.C $(A-B) \%$}} & \multirow{3}{*}{ hybridization } \\
\hline NBO & Occupancy & \multicolumn{2}{|c|}{$s \%$} & \multicolumn{2}{|c|}{$p \%$} & & & \\
\hline$(\mathrm{A}-\mathrm{B})$ bond & & A & B & A & $\begin{array}{c}\text { B } \\
\text { Pethidine }\end{array}$ & A & B & \\
\hline$\pi\left(\mathrm{C}_{3}-\mathrm{C}_{4}\right)$ & 1.663 & 0.00 & 0.00 & 100 & 100 & 49.99 & 50.01 & $0.7070\left(s p^{1}\right) \mathrm{C}+0.7072\left(s p^{1}\right) \mathrm{C}$ \\
\hline$\sigma\left(\mathrm{C}_{8}-\mathrm{O}_{9}\right)$ & 1.986 & 27.07 & 32.84 & 72.93 & 67.16 & 29.66 & 70.34 & $0.5446\left(s p^{2.69}\right) \mathrm{C}+0.8387\left(s p^{2.04}\right) 0$ \\
\hline$\pi\left(\mathrm{C}_{8}-\mathrm{O}_{10}\right)$ & 1.990 & 32.93 & 39.52 & 67.07 & 60.48 & 32.72 & 67.28 & $0.5720\left(s p^{2.04}\right) \mathrm{C}+0.8203\left(s p^{1.53}\right) 0$ \\
\hline$\sigma\left(\mathrm{C}_{14}-\mathrm{N}_{15}\right)$ & 1.980 & 24.09 & 28.97 & 75.91 & 71.03 & 38.35 & 61.65 & $0.6193\left(s p^{3.15}\right) \mathrm{C}+0.7851\left(s p^{2.45}\right) \mathrm{N}$ \\
\hline$\sigma\left(\mathrm{C}_{13}-\mathrm{C}_{14}\right)$ & 1.978 & 27.02 & 28.62 & 72.98 & 71.38 & 50.28 & 49.72 & $0.7091\left(s p^{2.70}\right) \mathrm{C}+0.7051\left(s p^{2.49}\right) \mathrm{C}$ \\
\hline & & & & & Diclofenac & & & \\
\hline$\pi\left(\mathrm{C}_{1}-\mathrm{C}_{2}\right)$ & 1.676 & 0.01 & 0.00 & 99.99 & 100 & 52.62 & 47.38 & $0.7254\left(s p^{99.99}\right) \mathrm{C}+0.6883\left(s p^{1}\right) \mathrm{C}$ \\
\hline$\sigma\left(\mathrm{C}_{17}-\mathrm{O}_{18}\right)$ & 1.991 & 25.93 & 32.53 & 74.07 & 67.47 & 29.23 & 70.77 & $0.5407\left(s p^{2.86}\right) \mathrm{C}+0.8412\left(s p^{2.07}\right) 0$ \\
\hline$\pi\left(\mathrm{C}_{17}-\mathrm{O}_{19}\right)$ & 1.993 & 34.21 & 37.54 & 65.79 & 62.46 & 33.45 & 66.55 & $0.5783\left(s p^{1.92}\right) \mathrm{C}+0.8158\left(s p^{1.66}\right) 0$ \\
\hline$\sigma\left(\mathrm{C}_{6}-\mathrm{N}_{7}\right)$ & 1.978 & 27.59 & 35.05 & 72.41 & 64.95 & 37.52 & 62.48 & $0.6125\left(s p^{2.62}\right) \mathrm{C}+0.7904\left(s p^{1.85}\right) \mathrm{N}$ \\
\hline$\sigma\left(\mathrm{C}_{9}-\mathrm{Cl}_{14}\right)$ & 1.981 & 20.65 & 19.02 & 79.35 & 80.98 & 42.18 & 57.82 & $0.6495\left(s p^{3.84}\right) \mathrm{C}+0.7604\left(s p^{4.26}\right) \mathrm{Cl}$ \\
\hline
\end{tabular}

S.P.C: Square of polarization co-efficient
Table 6. Second order perturbation theory of Fock matrix in NBO basis of pethidine and diclofenac drugs by B3PW91/DGDZVP

\begin{tabular}{|c|c|c|c|c|c|c|c|c|c|c|c|c|c|}
\hline \multirow[b]{2}{*}{ Donor (i) } & \multicolumn{4}{|c|}{ Pethidine } & \multirow[b]{2}{*}{$\mathrm{E}(\mathrm{i})-E(j)^{b}$} & \multirow[b]{2}{*}{$F(i, j)^{c}$} & \multicolumn{7}{|c|}{ Diclofenac } \\
\hline & Types & Acceptor (j) & Types & $E(2)^{a}$ & & & Donor (i) & Types & Acceptor (j) & Types & $E(2)^{a}$ & $\mathrm{E}(\mathrm{i})-E(j)^{b}$ & $F(i, j)^{c}$ \\
\hline $\mathrm{C}_{1}-\mathrm{C}_{2}$ & $\pi$ & $\mathrm{C}_{3}-\mathrm{C}_{4}$ & $\pi^{*}$ & 43.180 & 0.500 & 0.500 & $\mathrm{C}_{3}-\mathrm{C}_{4}$ & $\pi$ & $\mathrm{C}_{1}-\mathrm{C}_{2}$ & $\pi^{*}$ & 48.650 & 0.490 & 0.138 \\
\hline $\mathrm{C}_{8}-\mathrm{O}_{9}$ & $\pi$ & $\mathrm{C}_{7}-\mathrm{C}_{13}$ & $\pi^{*}$ & 2.660 & 1.760 & 0.061 & $\mathrm{C}_{5}-\mathrm{C}_{6}$ & $\pi$ & $\mathrm{C}_{3}-\mathrm{C}_{4}$ & $\pi^{*}$ & 48.870 & 0.500 & 0.140 \\
\hline $\mathrm{C}_{13}-\mathrm{C}_{14}$ & $\sigma$ & $\mathrm{N}_{15}-\mathrm{C}_{18}$ & $\sigma^{*}$ & 5.070 & 1.380 & 0.075 & $\mathrm{C}_{8}-\mathrm{C}_{9}$ & $\pi$ & $\mathrm{C}_{10}-\mathrm{C}_{11}$ & $\pi^{*}$ & 49.370 & 0.520 & 0.143 \\
\hline $\mathrm{LP}(2) \mathrm{O}_{9}$ & & $\mathrm{C}_{8}-\mathrm{O}_{10}$ & $\pi^{*}$ & 63.110 & 0.660 & 0.182 & $\mathrm{LP}(1) \mathrm{N}_{7}$ & & $\mathrm{C}_{5}-\mathrm{C}_{6}$ & $\pi^{*}$ & 27.960 & 0.580 & 0.120 \\
\hline $\mathrm{LP}(2) \mathrm{O}_{10}$ & & $\mathrm{C}_{7}-\mathrm{C}_{8}$ & $\sigma^{*}$ & 19.620 & 1.040 & 0.128 & $\mathrm{LP}(1) \mathrm{N}_{7}$ & & $\mathrm{C}_{8}-\mathrm{C}_{9}$ & $\pi^{*}$ & 31.820 & 0.530 & 0.123 \\
\hline $\mathrm{LP}(2) \mathrm{O}_{10}$ & & $\mathrm{C}_{8}-\mathrm{O}_{9}$ & $\sigma^{*}$ & 37.510 & 1.020 & 0.175 & $\mathrm{LP}(1) \mathrm{Cl}_{14}$ & & $\mathrm{C}_{8}-\mathrm{C}_{9}$ & $\pi^{*}$ & 11.50 & 0.590 & 0.082 \\
\hline $\mathrm{LP}(1) \mathrm{N}_{15}$ & & $\mathrm{C}_{14}-\mathrm{H}_{32}$ & $\sigma^{*}$ & 11.190 & 1.130 & 0.102 & $\mathrm{LP}(2) \mathrm{O}_{18}$ & & $\mathrm{C}_{17}-\mathrm{O}_{19}$ & $\pi^{*}$ & 50.74 & 0.690 & 0.169 \\
\hline $\mathrm{LP}(1) \mathrm{N}_{15}$ & & $\mathrm{C}_{16}-\mathrm{H}_{34}$ & $\sigma^{*}$ & 11.820 & 1.110 & 0.104 & $\mathrm{LP}(2) \mathrm{O}_{18}$ & & $\mathrm{C}_{17}-\mathrm{O}_{18}$ & $\pi^{*}$ & 42.99 & 0.960 & 0.182 \\
\hline
\end{tabular}

${ }^{\mathrm{a}} E^{2}=$ means energy of hyper conjugative interaction (stabilization energy), b energy difference between donor and acceptor $\mathbf{i}$ and $\mathbf{j}$ NBO orbitals. $F(\mathrm{i}, \mathrm{j})$ is the

Fock matrix element between i and j NBO orbitals 


\section{Conclusion}

In this research study, molecular structure and spectroscopy of pethidine and diclofenac drugs were investigated. Then, the FT-IR spectra were taken and the functional groups were determined and its normal vibrations were investigated. Energy gap and hybridization bonds, type of electron transfers between levels, natural atomic charge, and the amount of global hardness and global softness were calculated using the natural bond orbital (NBO) analysis at the presence of the solvents including $\mathrm{H}_{2} \mathrm{O}$, DMSO, $\mathrm{CH}_{3} \mathrm{CN}, \mathrm{C}_{3} \mathrm{H}_{6} \mathrm{O}, \mathrm{C}_{2} \mathrm{H}_{5} \mathrm{OH}$, and $\mathrm{CH}_{3} \mathrm{OH}$ on the optimized structure. The electronic transition properties which include the maximum excitation wavelength $\left(\lambda_{\max }\right)$ and relative intensities (oscillator strengths, $f$ ), Mulliken charges and natural population analysis were calculated using the B3LYP and (CIS-DFT) with GDZVP level of theory at the presence of six solvents. The ${ }^{1} \mathrm{H}$ and ${ }^{13} \mathrm{C}$ nuclear magnetic resonance (NMR), chemical shifts of pethidine, and diclofenac drugs were calculated using the Gauge-invariant atomic orbital (GIAO) method in the gas and liquid phases. Then, hybridization, Zero-point energy (ZPE), total energy $\left(E_{\mathrm{T}}\right)$, Dipole Moment $(m)$, polarizability $(\alpha)$, MEP, bond lengths, bond angles, electro negativity and ionization energy were calculated in the gas and liquid phases. The results indicated that, the computational method is a valuable method, providing invaluable information about the properties of the drugs. The results of the spectra depicted that the solvents had greater effect on the pethidine, and the medicinal effects of the pethidine is greater than that of the diclofenac.

\section{Acknowledgement}

The authors are grateful to all those who devote to the advancement of science specially professors in Gachsaran Islamic Azad university.

\section{Disclosure statement}

No potential conflict of interest was reported by the authors.

\section{ORCID}

Mostafa Khajehzadeh (D: 0000-0002-3841-9021

\section{References}

[1] R.F. Clark, E.M. Wei, P.O. Anderson. J. Emerge. Med., 1995, 13, 797-802.

[2] J.A. Fleet, M. Jones, I. Belan, Midwifery, 2017, 53, 15-19.

[3] A. Farina, G. Gostoli, E. Bossu, A. Montinaro, C. Lestingi, R. Lecce., J. Pharmaceut. Biomed., 2005, 37, 1089-1093.

[4] A.E. Elbohoty, H. Elrazek, M.A.E. Gawad, K.H.I. Abd-El-Maeboud, Int. J. Gynaecol. Obstet., 2012, 118, 7-10.

[5] Y. Huang, H. Zhang, C. Wei, G. Li, Q. Wu, J. Wang, Y. Song, Separat. Purificat. Technol., 2017, 172, 202-210.

[6] N.R. Lee, X. Zhang, M. Darna, L.P. Dwoskin, G. Zheng, Bioorg. Med. Chem. Lett., 2015, 25, 5032-5035.

[7] M.J. Wilson, C. MacArthur, C.A. Hewitt, K. Handley, F. Gao, L. Beeson, J. Daniels, R.T.C. Group, The Lancet., 2018, 392 (10148), 662-672.

[8] C. Jamey, B. Ludes, J.S. Raul, Toxicol. Anal. Clin., 2014, 26, 165-168.

[9] V. Tieppo Francio, S. Davani, C. Towery, T.L. Brown, J. Pain Palliat. Care Pharmacother., 2017, 31, 113-120.

[10] S. Shen, M.R. Marchick, Margaret R. Davis, George A. Doss, Lance R. Pohl, Chem. Res. Toxicol., 1999, 12, 214-222.

[11] S.J. Facey, B.A. Nebel, L. Kontny, M. Allgaier, B. Hauer, Environ. Tech. Innovat., 2018, 10, 55-61.

[12] D.R. Leenaraj, D. Manimaran, I. Hubert Joe, J. Mol. Struct., 2016, 1123, 180-190.

[13] A. Bhunia, P. Vojtíšek, V. Bertolasi, S.C. Manna, J. Mol. Struct., 2019, 1189, 94101. 
[14] B. Vijayakumar, V. Kannappan, V. Sathyanarayanamoorthi, J. Mol. Struct., 2016, 1121, 16-25.

[15] J. Juan, V. Orazi, M. Sandoval, P. Bechthold, A. Hernández-Laguna, C.I. Sainz-Díaz, E.A. González, M. Jenko, P.V. Jasen, Appl. Surface Sci., 2019, 489, 287-296.

[16] S. Hajlaoui, I. Chaabane, J. Lhoste, A. Bulou, K. Guidara, J. All. Compoun., 2016, 679, 302-315.

[17] Y.S. Mary, Y.S. Mary, K.S. Resmi, R. Thomas, Heliyo., 2019, 5, e02175.

[18] M. Szafran, A. Komasa, M. Anioła, A. Katrusiak, Z.D. Szafran, Vibrat. Spect., 2016, 84, 92-100.

[19] E. Mugunthan, M.B. Saidutta, P.E. Jagadeeshbabu, J. Photochem. Photobiol. Chem., 2019, 383, 111993.

[20] H. Al-Lawati, M.R. Vakili, A. Lavasanifar, S. Ahmed, F. Jamali, J. Pharm. Sci., 2019, 108, 2698-2707.

[21] M.J.E.A. Frisch, G.W. Trucks, H.B. Schlegel, G.E. Scuseria, M.A. Robb, J.R. Cheeseman, G. Scalmani, V. Barone, B. Mennucci, G. Petersson, H. Nakatsuji, Inc., Wallingford, CT, 200, 2009.
[22] M. Khajehzadeh, M. Moghadam, Spectrochim. Acta Part A. Mol. Biomol. Spect., 2017, 180, 51-66.

[23] M. Khajehzadeh, N. Sadeghi, J. Mol. Liq., 2018, 249, 281-293.

[24] M. Khajehzadeh, N. Sadeghi, J. Mol. Liq., 2018, 256, 238-246.

[25] M. Khajehzadeh, M. Rajabi, S. Rahmaniasl, J. Mol. Struct., 2019, 1175, 139-151.

[26] B. Amul, S. Muthu, M. Raja, S. Sevvanthi, J. Mol. Struct., 2019, 1195, 747-761.

[27] A.M. Fahim, M.A. Shalaby, M.A. Ibrahim, J. Mol. Struct., 2019, 1194, 211-226.

[28] S. Samiee, P. Hossienpour, Inorg. Chim. Acta., 2019, 494, 13-20.

[29] I.V. Mirzaeva, N.K. Moroz, I.V. Andrienko, E.A. Kovalenko, J. Mol. Struct., 2018, 1163, 68-76.

[30] K. Sharma, R. Melavanki, S.S. Patil, R. Kusanur, N.R. Patil, V.M. Shelar, J. Mol. Struct., 2019, 1181, 474-487.

[31] D.A. Zainuri, S. Arshad, N.C. Khalib, I.A. Razak, J. Mol. Struct., 2017, 1128, 520533.

[32] M.D. Mohammadi. M. Hamzehloo, Comput. Theo. Chem., 2018, 1144, 26-37.

[33] S. Bhunia, A. Kumar, A. Singh, A.K. Ojha., Comput. Theo. Chem., 2018, 1141, 7-14.

How to cite this manuscript: Mostafa Khajehzadeh, Sedigheh Rahmaniasl, Spectroscopic Behavior, FMO, NBO Analysis of Pethidine and Diclofenac Drugs by Theoretical Approach, Adv. J. Chem. A, 2020, 3(4), 391-407.

Copyright (C) 2020 by SPC (Sami Publishing Company)+ is an open access article distributed under the Creative Commons Attribution License, which permits unrestricted use, distribution, and reproduction in any medium, provided the original work is properly cited. 